\title{
Protective role of wogonin following traumatic brain injury by reducing oxidative stress and apoptosis via the PI3K/Nrf2/HO-1 pathway
}

\author{
YAN FENG $^{1}$, YARU JU ${ }^{2}$, ZHONGJIE YAN ${ }^{1}$, MINGJUN JI ${ }^{3}$, MING YANG ${ }^{1}$, \\ QIANG WU ${ }^{1}$, LIQUN WANG ${ }^{1}$ and GUOZHU SUN ${ }^{1}$ \\ ${ }^{1}$ Department of Neurosurgery, The Second Hospital of Hebei Medical University, Shijiazhuang, Hebei 050000; \\ ${ }^{2}$ Department of Obstetrics, The Fourth Hospital of Shijiazhuang, Shijiazhuang, Hebei 050011; \\ ${ }^{3}$ Department of Critical Care Medical, Linxi County People's Hospital, Xingtai, Hebei 054000, P.R. China
}

Received November 15, 2021; Accepted January 25, 2022

DOI: $10.3892 / \mathrm{ijmm} .2022 .5109$

\begin{abstract}
Traumatic brain injury (TBI) is usually caused by accidental injuries and traffic accidents, with a very high mortality rate. Treatment and management following TBI are essential to reduce patient injury and help improve long-term prognosis. Wogonin is a flavonoid compound with an antioxidant effect extracted from Scutellaria baicalensis Georgi. However, the function and mechanism of wogonin in protecting brain injury remain to be elucidated. The present study established a TBI model of Sprague-Dawley rats and treated them with wogonin following trauma. The results showed that wogonin treatment significantly reduced neurobehavioral disorders, brain edema and hippocampal neuron damage caused by TBI. It was found that in TBI rats, administration of wogonin increased the levels of antioxidant factors glutathione, superoxide dismutase and catalase in the CA1 region of the hippocampus and significantly inhibited the production of malondialdehyde and reactive oxygen species. western blotting data showed that wogonin exerted antioxidant activity by downregulating the level of $\mathrm{NOX}^{2}$ protein. In inhibiting cell apoptosis, wogonin upregulated the expression of Bcl-2 protein in the hippocampal CA1 region of TBI rats and inhibited caspase- 3 and Bax proteins. Additionally, wogonin inhibited the progression of injury following TBI through the PI3K/Akt/nuclear factor-erythroid factor 2-related factor 2 (Nrf2)/heme oxygenase-1 (HO-1) signaling pathway. Wogonin increased the expression of phosphorylated Akt, Nrf2 and HO-1 in the hippocampus of TBI rats. Following the administration of PI3K inhibitor LY294002, the upregulation
\end{abstract}

Correspondence to: Dr Guozhu Sun, Department of Neurosurgery, The Second Hospital of Hebei Medical University, 215 Heping West Road, Shijiazhuang, Hebei 050000, P.R. China

E-mail: sunguozhu705@126.com

Key words: wogonin, traumatic brain injury, oxidative stress, apoptosis, PI3K/Nfr2/HO-1 of these proteins by wogonin was partly reversed. In addition, LY294002 partially reversed the regulation of wogonin on $\mathrm{NOX}^{2}$, caspase-3, Bax and Bcl-2 proteins. Therefore, wogonin exerts antioxidant and anti-apoptotic properties to prevent hippocampal damage following TBI, which is accomplished through the PI3K/Akt/Nrf2/HO-1 pathway.

\section{Introduction}

Traumatic brain injury (TBI) has the highest mortality and disability rate of all types of body trauma. It is usually caused by accidental injuries and traffic accidents and 10 million new patients are affected each year worldwide (1). According to the pathogenic mechanism, TBI can be divided into primary ischemic brain injury and secondary injury. Primary brain tissue injury and intracranial hemorrhage are irreversible, in which inflammation, brain edema and neuronal apoptosis usually begin to appear several $\mathrm{h}$ after the injury. However, secondary damage is a pathological process that gradually develops following TBI and subsequent damage can be reduced by effective therapy $(2,3)$. Therefore, exploring the mechanism of secondary injury following TBI and finding clinically effective interventions following TBI will help the prognosis of patients.

Apoptosis, inflammatory storm, mitochondrial dysfunction and destruction of the blood-brain barrier, which ultimately leads to further brain tissue necrosis, are several aspects of the secondary injury that occurs following TBI (4). Mitochondrial dysfunction results in a large number of reactive oxygen species (ROS), free radicals and malondialdehyde (MDA) released in large enough quantities that the local expression of antioxidants such as superoxide dismutase (SOD) is not enough to completely counterbalance their damage $(5,6)$. ROS and MDA destroy nerve cell membranes, promote lipid peroxidation and chromatin damage and aggravate oxidative stress. For example, nicotinamide adenine dinucleotide phosphate (NADP) oxidase $\mathrm{NOX}^{2}$ causes oxidative damage to neurons following brain trauma by increasing the production of ROS (7). In addition, nuclear factor-erythroid 2 related factor 2 (Nrf2) mediates tissue resistance to oxidative stress. Combined antioxidant therapy to inhibit $\mathrm{NOX}^{2}$ and activate 
Nrf2 decreases secondary brain damage and improves functional recovery following TBI (8). The use of pramipexole (D2-like receptor agonist) to activate the transcription process of Nrf2 and its downstream detoxification enzyme heme oxygenase-1 (HO-1) has a significant neuroprotective effect following TBI (9). Therefore, it is important to determine if compounds with antioxidant properties, such as wogonin, a flavonoid compound extracted from the plant Scutellaria baicalensis Georgi and used in traditional Chinese medicine, are effective treatments for reducing secondary TBI injury.

Wogonin has a variety of biological activities including anti-inflammatory, antioxidant and anti-tumor properties (10-12). For example, wogonin alleviates tubulointerstitial fibrosis and kidney tubular epithelial injury through the PI3K/Akt/NF- $\kappa \mathrm{B}$ signaling pathway that mediates autophagy and inflammation in diabetic nephropathy (13). In a cell model of lung injury, wogonin prevents apoptosis of injured lung cells by inhibiting the expression of the Bcl-2 antagonist of cell death (BAD) gene and subsequent activation of cleaved caspase-3 through the PI3K/AKT pathway (14). In the prevention of TBI, wogonin attenuates the symptoms of TBI in Wistar rats caused by fluid shock injury (15). In addition, wogonin treatment improves long-term functional and histological outcomes, reduces brain edema and attenuates the Toll-like receptor (TLR)4/NF-кB-mediated inflammatory response in mouse TBI (16). Despite evidence indicating the benefits of wogonin treatment to neurological recovery in stroke models, the precise mechanisms of these protective effects remain to be elucidated. In particular, it is unclear whether wogonin exerts cognitive enhancing or preservation effects by attenuating oxidative stress and apoptosis in TBI and whether it regulates $\mathrm{PI} 3 \mathrm{~K} / \mathrm{Nrf} 2 / \mathrm{HO}-1$ signaling in the brain.

The present study revealed that wogonin exhibited neuroprotective effects in a rat model of TBI. In terms of mechanism, wogonin activated $\mathrm{Nrf} 2 / \mathrm{HO}-1$ signaling in a PI3K/Akt-dependent manner and attenuated oxidative stress and apoptosis in the hippocampus of rats following TBI. The present study provided a new basis for drug treatment and neuroprotection following TBI.

\section{Materials and methods}

Establishment of TBI model. A total of 120 adult male SD rats (body weight 300-330 g; aged 10-12 weeks; purchased from animal Experiment Center of Hebei Medical University) were provided with food and water ad libitum and kept in a specific pathogen-free (SPF) animal room under controlled conditions (12-h light/dark cycle and $22 \pm 3{ }^{\circ} \mathrm{C}$ temperature; humidity $40-70 \%)$. The TBI model is induced by the controlled cortical impingement (CCI) method, as described previously (17). In short, rats anesthetized with $10 \%$ chloral hydrate $(300 \mathrm{mg} / \mathrm{kg}$, intraperitoneally) were fixed in a stereotactic frame. Rats were anesthetized $\sim 3$ min following injection and all animals exhibited no side effects, such as peritonitis, pain, or discomfort. A midline incision was made to expose the skull and then a $6.0 \mathrm{~mm}$ diameter right-hand hole was made through a surgical electric skull grinder. The opening was centered on the coronal suture and $2.5 \mathrm{~mm}$ outside the sagittal suture. An electromagnetic impactor (round tip diameter $4 \mathrm{~mm}$; speed $5 \mathrm{~m} / \mathrm{sec}$; residence time $100 \mathrm{msec}$ ) was used to inflict CCI injury on rats with an impact depth of $2.0 \mathrm{~mm}$. Finally, the bone flap was sealed and sutured. For the sham operation group, the animals received the same anesthesia and surgical scalp incision, but no impact injury. All animal experiments were approved by the Ethics Committee of Hebei Medical University (permit no. 20201086). Humane endpoints were established following the guideline of assessment for humane endpoints in animal experiment [People's Republic of China: RB/T 173-2018; bingdian001.com (lascn.net)] in order to minimize pain or distress to experimental animals. After the breathing of the rat became slow and weak and it did not respond to stimulation, the rats was sacrificed by euthanasia under anesthesia (sodium pentobarbital $40 \mathrm{mg} / \mathrm{kg}$, intraperitoneally). Cervical vertebra dislocation was used for euthanasia and death was confirmed by cessation of the heartbeat.

Groups and drug administration. The rats were randomly assigned into three groups: sham operation group, TBI model group and wogonin treatment group following TBI. Each sub-group was composed of five rats, testing after injury was as follows: i) Neurological severity scores at days 1, 3, 5, 7 and 14 ; ii) determination of brain water content at days 3 ; iii) Morris Water Maze (MWM) test at days 3-7; and iv) histological analysis, TUNEL, ROS and antioxidant enzymes activity and western blot analysis at day 1, 3, 7. Wogonin (MilliporeSigma) was prepared by dissolving in $25 \%$ dimethyl sulfoxide (DMSO). According to the dose provided in a previous study (16), wogonin solution $(40 \mathrm{mg} / \mathrm{kg}$ ) was injected intraperitoneally at $10 \mathrm{~min}, 24$ and $48 \mathrm{~h}$ after the surgery. The sham group and TBI group were both intraperitoneally injected with the same amount of normal saline. Sham-operated rats underwent procedures identical to those of the TBI animals, including anesthesia and craniotomy surgery, but without TBI. It should be noted that no adverse effects or mortality were observed in rats treated with wogonin during the experiments. Therefore, from the perspective of animal welfare, a blank control group was not established.

For the study of the mechanism of wogonin affecting the hippocampus, rats were randomly divided into 5 groups: Sham operation group, TBI model group, wogonin treatment group following TBI, wogonin and LY294002 treatment group following TBI and DMSO administration control following TBI. PI3K inhibitor LY294002 was dissolved in 25\% DMSO in PBS at the time of administration. Rats were infused with $50 \mathrm{mM}$ LY294002 in one side of the cerebral ventricle $30 \mathrm{~min}$ before TBI. Specifically, according to the previous study (18), rats under anesthesia were provided with stepping electric micro-injectors (Stoelting) to inject LY294002 or DMSO into the left cerebral ventricle at a rate of $1 \mu \mathrm{l} / \mathrm{min}$. A total of 120 rats were used in the present study, of which 2 rats that received CCI died of brain damage.

Neurological severity score. The neurological severity score (NSS) is a composite of motor, sensory, reflex and balance tests before and after treatment in rats, as described previously (19). All rats were assessed for NSS on days 1, 3, 5, 7 and 14 following TBI. Scores are evaluated by researchers who are unaware of the grouping, with 1 point for inability to perform each test or lack of test reflexes. Normal rats have a score of 2-3, while the maximum injury score is 18 . 
Evaluation of cerebral edema. The degree of cerebral edema is assessed by relative brain water content analysis, as described previously (20). Rats were sacrificed by decapitation under anesthesia ( $2 \%$ sodium pentobarbital, $40 \mathrm{mg} / \mathrm{kg}$, i.p. injected) on the 3rd day following TBI or sham operation. The rat brain was harvested and immediately weighed as wet weight (WW). The brain tissue was then dried at $100^{\circ} \mathrm{C}$ for $24 \mathrm{~h}$ to obtain a dry weight (DW). The relative brain water content is calculated as follows: (WW-DW)/WW x100\%.

Morris water maze (MWM). According to previous research (21), the spatial learning and memory ability of rats was evaluated in the MWM test 3-7 days following TBI. Prior to the test, the rats swam freely in the circular pool for $5 \mathrm{~min}$. In the central area of the target quadrant, a $2 \mathrm{~cm}$ opaque hidden platform (12 cm in diameter and $28 \mathrm{~cm}$ in height) was set up. Prior to the operation, all the rats were guided to the target platform. In each test, $60 \mathrm{sec}$ were set aside for the rat to find the platform. The camera hanging above the maze was connected to a video tracking system (HVS Imaging) and the escape delay time was recorded. The average escape latency of four repeated trials was calculated. On the last day of the experiments, the hidden platform was removed and the percentage of time the rat stayed in the target quadrant tested. The average swimming speed of rats in each group was also recorded.

Hematoxylin and eosin staining $(H \& E)$. The brain tissues were fixed in $4 \%$ paraformaldehyde solution for $24 \mathrm{~h}$ at room temperature, washed with running water for $4 \mathrm{~h}$, then dehydrated with graded alcohol and embedded in paraffin following standard histological procedures. The paraffin tissue was sectioned at $4 \mu \mathrm{m}$. Sample sections were stained with $H \& E$ according to the usual protocol (hematoxylin for $2 \mathrm{~min}$ and eosin for $30 \mathrm{secs}$ at room temperature). Images of the staining results of the hippocampal brain slices were captured under a light microscope (BX51; Olympus Corporation; magnification $\times 50$ and $\times 200$ ) and the pyramidal cell density (number $/ \mathrm{mm}^{2}$ ) in the CA1 area was calculated. Cell counts from hippocampus CA1 on each of the four sections were averaged to provide a single value (number of neurons $/ \mathrm{mm}^{2}$ ) for each animal. Image Pro Plus 6.0 software (Media Cybernetics) was used for analysis.

Cell apoptosis assay. According to the manufacturer's instruction, the TdT-mediated dUTP nick-end labeling (TUNEL) apoptosis detection kit (cat. no. C1088, Beyotime Institute of Biotechnology) was used to detect neuronal apoptosis in the hippocampus. Briefly, the hippocampal tissue sections of each group of rats were deparaffinized and rehydrated. Then the sections were incubated with $10 \mu \mathrm{g} / \mathrm{ml}$ proteinase $\mathrm{K}$ solution at $37^{\circ} \mathrm{C}$ for $15 \mathrm{~min}$. The sections were stained with green fluorescein-labeled dUTP solution for $10 \mathrm{~min}$ at room temperature. Finally, the section was sealed in a mounting medium containing DAPI. The number of TUNEL-DAPI positive cells was counted using a fluorescence microscope (Olympus Fluoview FV1000; Olympus Corporation; magnification $\mathrm{x} 200$ ) according to a previous study (22). Image Pro Plus 6.0 software (Media Cybernetics) was used for analysis.
Immunohistochemistry IHC. The brain tissue sections of TBI rats and wogonin treatment rats were used for IHC detection. The paraffin sections were incubated with $3 \% \mathrm{H}_{2} \mathrm{O}_{2}$ for $20 \mathrm{~min}$ at room temperature and then blocked with goat serum (cat. no. C0265; Beyotime Institute of Biotechnology) for $1 \mathrm{~h}$ at room temperature. Then the sections were incubated with $\mathrm{NOX}^{2}$ primary antibody (1:200 dilution; Abcam; cat. no. ab80508) at $4^{\circ} \mathrm{C}$ overnight. Following washing with PBS, the sections were incubated with goat anti-rabbit IgG-HRP secondary antibody (1:500 dilution; cat. no. sc-2030; Santa Cruz Biotechnology, Inc.) for $1 \mathrm{~h}$ at room temperature. After counterstaining with hematoxylin, images of the sections were captured using the AxioVision4Ac microscope system (Carl Zeiss AG). IHC images were analyzed with optical density (OD) value by ImageJ software v1.41 (National Institutes of Health). The deeper the DAB staining, the greater the OD value and protein expression.

NOX activity determination. NOX activity analysis was carried out according to previous research (23). The hippocampal tissue samples were homogenized and lysed and then centrifuged at $1,000 \mathrm{xg}$ for $10 \mathrm{~min}$ at $4^{\circ} \mathrm{C}$ to collect the supernatant. The supernatant was centrifuged in an ultracentrifuge at $13,000 \mathrm{x} \mathrm{g}$ at $4^{\circ} \mathrm{C}$ for $20 \mathrm{~min}$ to separate the membrane components and $50 \mu \mathrm{g}$ membrane fraction was used to determine NOX enzyme activity. Relative light units (RLU) were recorded every $1 \mathrm{~min}$ continuously for $5 \mathrm{~min}$ by a standard luminometer, as the indicator of NOX activity (RLU/ $\mu \mathrm{g} / \mathrm{min}$ ).

The activities of SOD, catalase (CAT), glutathione (GSH) and the levels of MDA and ROS measurement. The hippocampal tissue following TBI or administration was mechanically homogenized and then $0.5-10 \%$ of the tissue homogenate was used to detect oxidative stress-related indicators. The measurement of SOD activity was carried out in accordance with the manufacturer's instructions (cat. no. A001; Nanjing Jiancheng Bioengineering Institute). The SOD level was measured by the absorbance of the sample at $550 \mathrm{~nm}$, expressed as a unit/g protein. CAT activity was measured by ammonium molybdate spectrophotometry according to the manufacturer's instructions (cat. no. A007; Nanjing Jiancheng Bioengineering Institute). CAT decomposed the hydrogen peroxide in the tissue and then the remaining hydrogen peroxide reacted with ammonium molybdate to form a yellow complex. The activity of CAT was evaluated by measuring the content of the yellow complex. Tissue MDA was determined by using the thiobarbituric acid (TBA) reaction method as described previously (7). MDA reacted with TBA to form a pink chromogen and its absorbance at $532 \mathrm{~nm}$ could represent the MDA content (nmol $\mathrm{MDA} / \mathrm{g}$ tissue). For the measurement of GSH, the principle is that 5,5'-dithiobis-2-nitrobenzoic acid oxidizes GSH in tissues to produce 5-thio-2-nitrobenzoic acid. The GSH measurement was performed according to the instructions of the manufacturer of the kit (cat. no. 703002; Cayman Chemical Company). The level of ROS was carried out in accordance with the manufacturer's instructions (cat. no. E004 Nanjing Jiancheng Bioengineering Institute). The hippocampal tissue homogenate was centrifuged at $500 \mathrm{xg}$ for $10 \mathrm{~min}$ at $4^{\circ} \mathrm{C}$ and then $1 \mathrm{ml}$ of $0.01 \mathrm{M}$ PBS solution was used to resuspend the cell pellet. $10 \mu \mathrm{l}$ $1 \mathrm{mM}$ 2,7-dichlorodihydrofluorescein diacetate was added to 
the cells. After incubating for $30 \mathrm{~min}$ at $37^{\circ} \mathrm{C}$, the fluorescence intensity was quantified using a spectrofluorometer (excitation, $500 \mathrm{~nm}$; emission, $525 \mathrm{~nm}$ ).

Immunofluorescence. Briefly, after intracardially perfused with $4 \%$ paraformaldehyde solution for $30 \mathrm{~min}$ at $4^{\circ} \mathrm{C}$, brains tissues were removed, post-fixed in the same fixative for 1 day at room temperature and subsequently soaked in $30 \%$ sucrose for 2-3 days at $4^{\circ} \mathrm{C}$. Tissues were embedded in an optimal cutting temperature compound (OCT, Sakura Finetek Europe B.V.) and frozen sections cut at $15 \mu \mathrm{m}$. The frozen sections were permeabilized with $0.4 \%$ Triton X-100 for $10 \mathrm{~min}$ and then blocked with $10 \%$ donkey serum (cat. no. ab138579; Abcam) for $30 \mathrm{~min}$ at room temperature. Sections with NeuN antibody (dilution 1:100; cat. no. MAB377; MilliporeSigma), phosphorylated (p-)Akt antibody (dilution 1:100; cat. no. PA5-104867; Invitrogen; Thermo Fisher Scientific, Inc.) or Nrf2 antibody (dilution 1:100; cat. no. sc-722; Santa Cruz Biotechnology, Inc.) were incubated overnight at $4^{\circ} \mathrm{C}$. The sections were incubated with fluorescently-labeled secondary antibody Alexa-Fluor594 or Alexa-Fluor 488 (diluted 1:200; cat. no. sc-362281 and sc-362257; Santa Cruz Biotechnology, Inc.) for $2 \mathrm{~h}$ in the dark. Finally, the slices were observed and images captured under a fluorescence microscope (Olympus Fluoview FV1000; Olympus Corporation; magnification, $\mathrm{x} 200$ ). The counting area was located in the same position in all groups, and the number of p-Akt-positive or Nrf2-positive $\mathrm{NeuN}$ in the hippocampus CA1 was counted for each section. For each group, quantification was performed in sections from five different rats.

Western blotting. The hippocampus tissue of the rats in the TBI group or the administration group was lysed in RIPA lysis buffer (Thermo Fisher Scientific, Inc.) and the protein concentration was quantified by BCA reagent (Beijing Solarbio Science \& Technology Co., Ltd. ). Protein $(30 \mu \mathrm{g})$ was used for $12 \%$ SDS-PAGE electrophoresis and then transferred to PVDF membrane (Bio-Rad Laboratories, Inc.). The membrane was blocked with $5 \%$ skimmed milk for $2 \mathrm{~h}$ at room temperature and then incubated with the primary antibodies overnight at $4^{\circ} \mathrm{C}$. The primary antibodies used included: $\mathrm{NOX}^{2}$ antibody (dilution 1:500; cat. no. ab80508; Abcam), Nrf2 antibody (dilution 1:500; cat. no. sc-722; Santa Cruz Biotechnology, Inc.), p-Akt antibody (dilution 1:500; PA5 -104867; Invitrogen), Akt antibody (dilution 1:500; cat. no. MA5-14898; Invitrogen), caspase-3 antibody (dilution 1:500; cat. no. sc-56053; Santa Cruz Biotechnology, Inc.), Bax antibody (dilution 1:500; cat. no. sc-65532; Santa Cruz Biotechnology, Inc.), Bcl-2 antibody (dilution 1:500; cat. no. sc-7382; Santa Cruz Biotechnology, Inc.), HO-1 antibody (dilution 1:500; cat. no. sc-136960; Santa Cruz Biotechnology, Inc.) and $\beta$-actin antibody (dilution 1:1,000; cat. no. AF7018; Affinity Biosciences). The membrane was then incubated with horseradish peroxidase (HRP)-conjugated secondary antibody [goat anti-rabbit IgG-HRP (cat. no. sc-2030); goat anti-mouse IgG-HRP (cat. no. sc-2005). 1:2,000 dilution; Santa Cruz Biotechnology, Inc.] for $2 \mathrm{~h}$. The western blotting signal on the membrane was obtained by an enhanced chemiluminescence (ECL) detection system. ImageJ software v 4.1 (National Institutes of Health) was used to quantify the optical density signal.
Statistical analysis. SPSS 16.0 software (SPSS, Inc.) was used for data analysis, and all data were expressed as mean \pm standard deviation (SD). Data comparison among multiple groups was conducted with one-way analysis of variance (ANOVA), statistical analysis between two groups were performed with the Newman-Keuls test and between multiple groups with one-way ANOVA followed by Tukey test. $\mathrm{P}<0.05$ was considered to indicate a statistically significant difference.

\section{Results}

Wogonin attenuates neurological damage following traumatic brain injury. The present study established a rat TBI model and verified if wogonin alleviated the neurological damage and cognitive function caused by TBI. First, the NSS assessment and the degree of cerebral edema were used to evaluate the neurological deficit. Compared with sham-operated animals, TBI at 1-14 days significantly increased the NSS score of rats $\left({ }^{*} \mathrm{P}<0.05\right)$. After treatment with wogonin in TBI rats, wogonin significantly reduced the NSS score on days 3, 5, 7 and $14\left({ }^{\#} \mathrm{P}<0.05\right.$, Fig. 1A). Brain water content analysis was used to evaluate the therapeutic effect of wogonin on brain edema in TBI model. TBI induced a significant increase in brain water content $\left({ }^{*} \mathrm{P}<0.05\right)$, which could be alleviated by wogonin treatment ( ${ }^{\#} \mathrm{P}<0.05$, Fig. 1B). Next, the MWM test was used to evaluate whether wogonin helps to alleviate the spatial memory impairment induced following TBI. As shown in Fig. 1C, TBI caused a significant spatial learning deficit compared with the rats in the sham group and TBI rats spent a longer time searching for the hidden platform at 3-7 days post-surgery $\left({ }^{*} \mathrm{P}<0.05\right)$. However, rats in the wogonin group displayed a profoundly shorter latency time at 4-7 days as compared to those in the TBI group $\left({ }^{\#} \mathrm{P}<0.05\right)$. After removing the hidden platform, TBI caused the rat to spend an extended time in finding the target quadrant that previously contained the platform. The administration of wogonin significantly shortened the time for TBI model mice to navigate to the target quadrant (Fig. 1D). During this period, there was no significant difference in the swimming speed of the rats in each group (Fig. 1E), which proved that the time they searched for the target was not affected by their swimming speed. These results prove that wogonin slows down the neurological damage and reduces brain edema following TBI in rats and helps to improve the learning and memory ability.

$H \& E$ staining was used to measure hippocampal neurons densities to determine if the behavior in the water maze correlated with brain recovery of rats treated with wogonin following TBI. The H\&E staining results showed that there was significant neuronal damage in the hippocampus of rats with TBI, which was manifested as nuclear pyknosis, decreased number of neurons and neuron atrophy. However, wogonin treatment reduced neuronal loss caused by TBI (Fig. 2A). The statistical results of the number of neurons in the stained visual field showed that TBI significantly induced the death of pyramidal cells in the hippocampus $24 \mathrm{~h}$ after injury, while wogonin prevented the loss of neurons induced by TBI ( ${ }^{*} \mathrm{P}<0.05$ vs. sham group; ${ }^{\#} \mathrm{P}<0.05$ vs. TBI group; Fig. $\left.2 \mathrm{~B}\right)$. These results proved that wogonin reduces the damage of hippocampal neurons induced by TBI. 

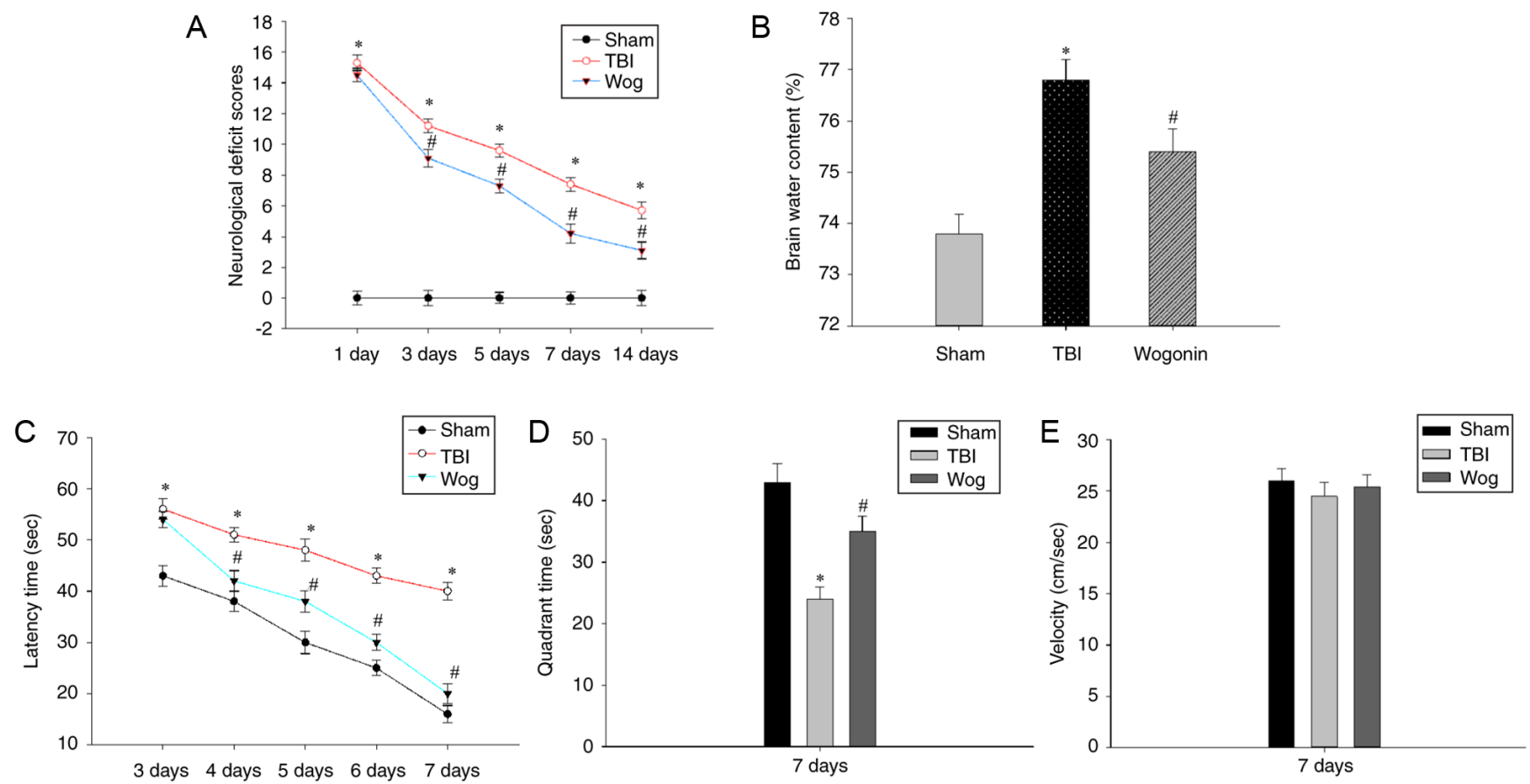

Figure 1. Wogonin treatment following TBI improved neurobehavioral function and reduced cerebral edema. (A) The neurological severity scores of rats in the sham operation group (sham), TBI model group (TBI) and wogonin administration group (Wog) were recorded on the 1,3,5,7 and 14 days after the operation. (B) The brain water content of the three groups of rats on the 3rd day was tested. (C) In the Morris water maze test, the latency time of the three groups of rats looking for a hidden platform was recorded. (D) On the 7th day, the three groups of rats were tested in the quadrant time of the target quadrant of the removal platform. (E) The swimming speed of the three groups of rats was tested. Data are expressed as mean \pm standard deviation ( $\mathrm{n}=5 /$ group; ${ }^{*} \mathrm{P}<0.05 \mathrm{vs}$. sham group; ${ }^{\text {"}} \mathrm{P}<0.05$ vs. TBI group). TBI, traumatic brain injury.

A

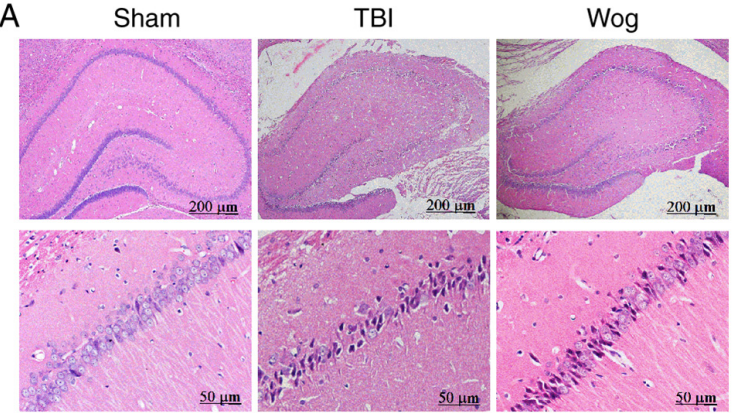

B

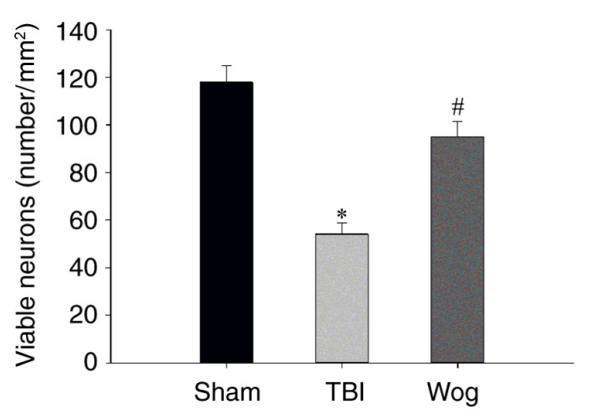

Figure 2. Wogonin reduces hippocampal neuron damage following TBI. (A) Representative hematoxylin and eosin staining images of the hippocampal CA1 area showed sham operation group (sham), TBI model group (TBI) and wogonin administration group (Wog) within $24 \mathrm{~h}$. Scale bars: 200 and $50 \mu \mathrm{m}$. (B) The number of viable neurons $\left(\mathrm{mm}^{2}\right)$ in the hippocampus of each group was counted ( $\mathrm{n}=5 /$ group; ${ }^{*} \mathrm{P}<0.05$ vs. sham group; ${ }^{*} \mathrm{P}<0.05$ vs. TBI group). TBI, traumatic brain injury.

Wogonin promotes anti-oxidation following TBI. In order to explore the mechanism of wogonin in alleviating the neurological damage caused by TBI, its role in anti-oxidation was first tested. The levels of GSH, SOD, CAT, MDA and ROS in hippocampal tissues 1,3 and 7 days after the operation were used as detection indicators. The results showed that TBI caused a significant decrease in the levels of GSH, SOD and CAT in the hippocampus. However, administration of wogonin following TBI increased the amount of these antioxidant factors $\left({ }^{*} \mathrm{P}<0.05\right.$ vs. TBI group; Fig. 3A-C). In addition, compared with the TBI model group, the administration of wogonin significantly reduced the levels of oxidative stress products MDA and ROS ( $\mathrm{P}<0.05$ vs. TBI group; Fig. 3D-E). These results proved that wogonin had a repairing effect through anti-oxidation in the hippocampus damaged following TBI.

Since NADPH oxidase mediates oxidative stress-related damage, the expression of $\mathrm{NOX}^{2}$, the main type of NADPH oxidase, was examined by IHC and western blotting. As shown in Fig. 4A, occasional $\mathrm{NOX}^{2}$ positive cells were detected on day 3 in the sham group. By contrast, $\mathrm{NOX}^{2}$ positive cells were significantly increased 3 days following TBI as indicated with an intense color due to enhanced immune reactivity. However, the immune reactivity of $\mathrm{NOX}^{2}$ in the wogonin group was significantly weaker than that in the TBI group (Fig. 4B). Compared with the TBI model group, the administration of wogonin significantly reduced the enzyme activity of NOX in the hippocampal CA1 region on days 1,3 and 7 ( $\mathrm{P}<0.05$ vs. sham group; ${ }^{*} \mathrm{P}<0.05$ vs. TBI group; Fig. $\left.4 \mathrm{C}\right)$. western blotting was used to detect protein expression in hippocampal CA1 area on day 1, 3 and 7 after the operation (Fig. 4D). The results of blotting optical density analysis showed that, compared with the sham group, the $\mathrm{NOX}^{2}$ protein expression in the TBI group was significantly upregulated at 1,3 and 7 days after the 
A

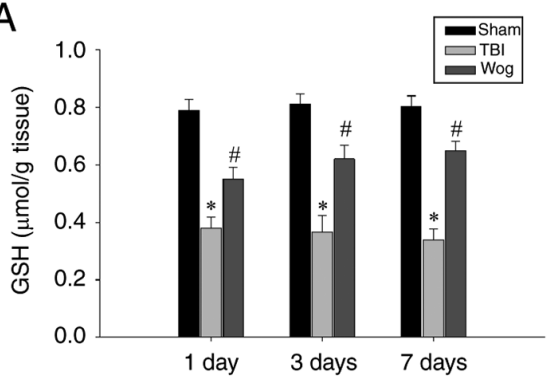

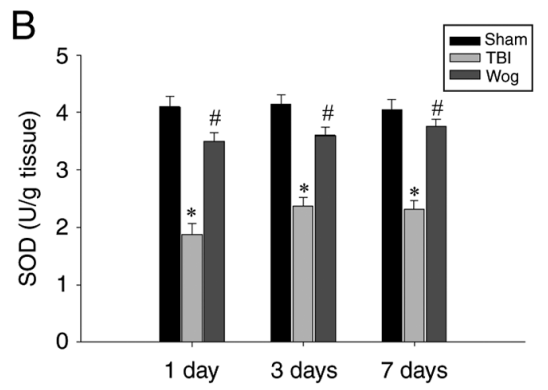
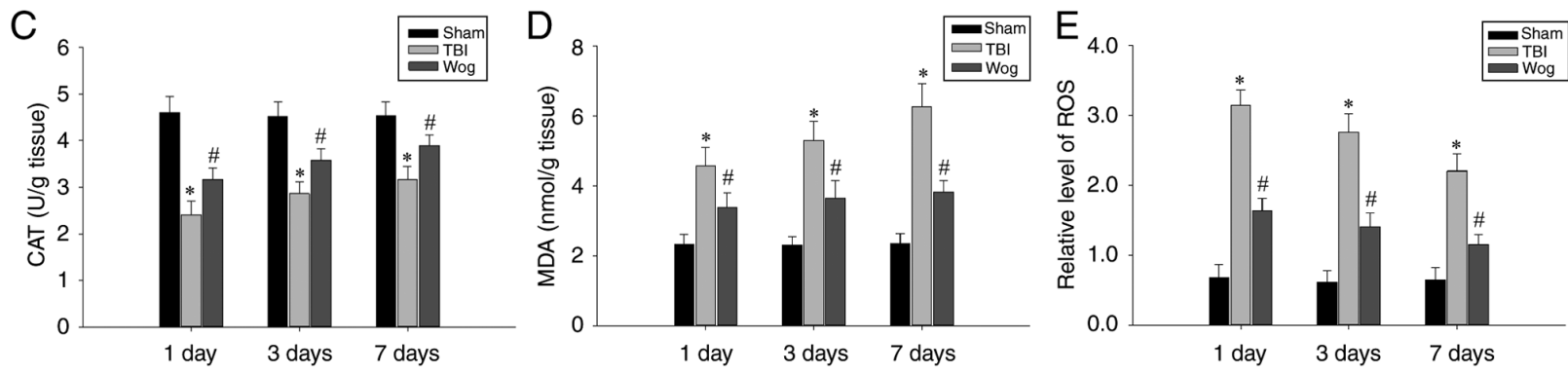

Figure 3. Wogonin regulates the levels of oxidative stress-related factors. The levels of (A) GSH, (B) SOD, (C) CAT, (D) MDA and (E) ROS in the hippocampus of rats in the sham operation group (sham), TBI model group (TBI) and wogonin administration group (Wog) were detected on the 1, 3 and 7 days after the operation. Data are expressed as mean \pm standard deviation ( $\mathrm{n}=5 /$ group; ${ }^{*} \mathrm{P}<0.05$ vs. sham group; ${ }^{*} \mathrm{P}<0.05$ vs. TBI group). GSH, glutathione; SOD, superoxide dismutase; CAT, catalase; MDA, malondialdehyde; ROS, reactive oxygen species; TBI, traumatic brain injury.

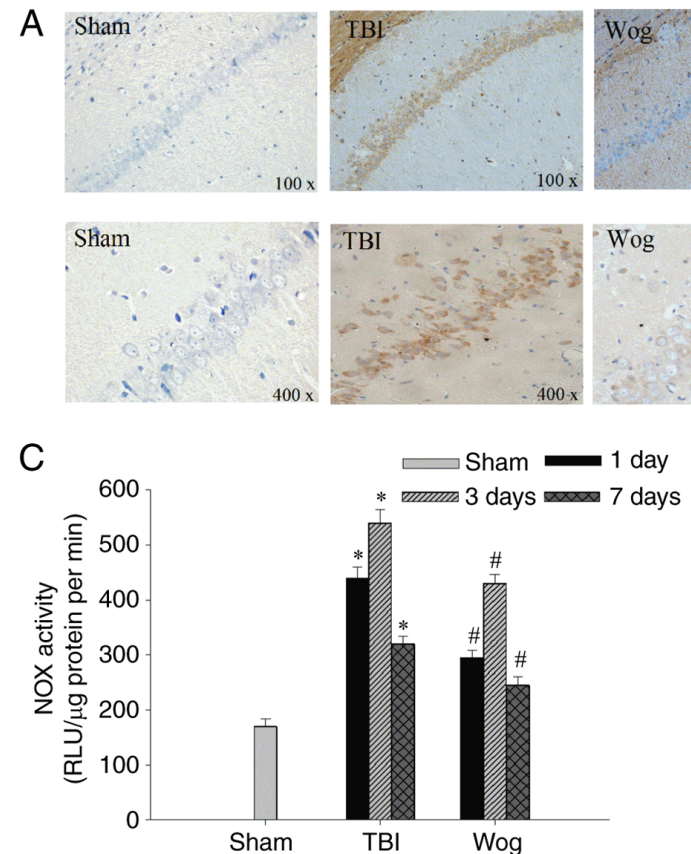

$\mathrm{E}$

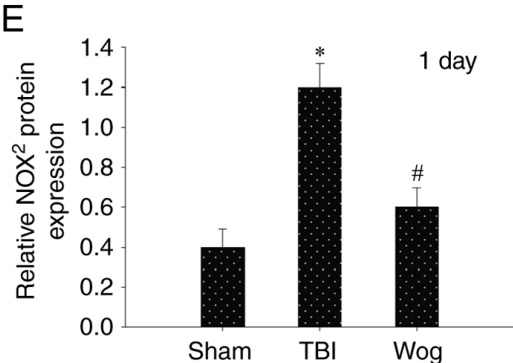

$\mathrm{F}$

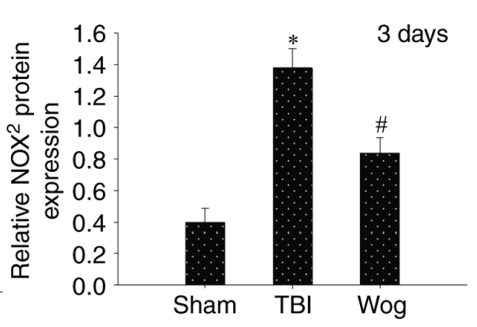

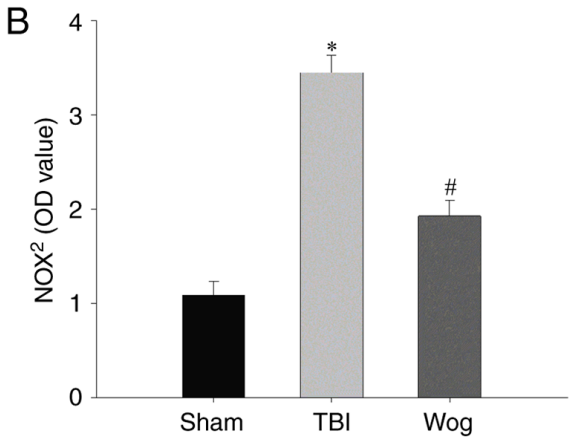

$\mathrm{D}$

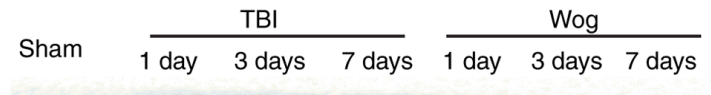

$\beta$-actin

$\mathrm{G}$

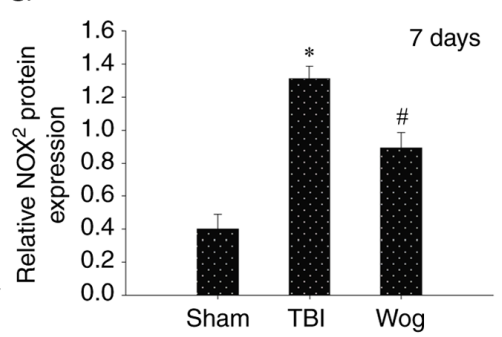

Figure 4. Wogonin regulates the protein expression of $\mathrm{NOX}^{2}$. (A) Representative immunohistochemistry staining of NOX ${ }^{2}$ in hippocampal CA1 area of the sham operation group (sham), TBI model group (TBI) and wogonin administration group (Wog) at 3 days. (B) Quantitative analysis of NOX ${ }^{2}$ in the hippocampus. (C) NOX activity was detected in the hippocampus of TBI or sham-operated rats on days 1,3 and 7. (D) Western blotting of NOX ${ }^{2}$ protein level in hippocampal CA1 area was performed at 1,3 and 7 days after the operation. The OD of the $\mathrm{NOX}^{2}$ band at (E) 1, (F) 3 and (G) 7 days relative to $\beta$-actin were analyzed. Data are expressed as mean \pm standard deviation ( $\mathrm{n}=5$ /group). ${ }^{*} \mathrm{P}<0.05$ vs. sham group; ${ }^{*} \mathrm{P}<0.05$ vs. TBI group. TBI, traumatic brain injury; OD, optical density, RLU, relative light units. 
operation. Compared with the TBI group, the administration of wogonin significantly reduced the expression of $\mathrm{NOX}^{2}$ protein $\left({ }^{*} \mathrm{P}<0.05\right.$ vs. sham group; ${ }^{\#} \mathrm{P}<0.05$ vs. TBI group; Fig. $\left.4 \mathrm{E}-\mathrm{G}\right)$. Therefore, wogonin exercised its antioxidant function by decreasing the expression of $\mathrm{NOX}^{2}$ protein.

Wogonin inhibits apoptosis of hippocampal cells induced by TBI. TUNEL assay was used to evaluate the apoptosis in the CA1 area of the hippocampus following surgery (Fig. 5A). The statistical results showed that TBI significantly increased the number of TUNEL-positive cells in the CA1 region of the hippocampus. However, compared with the TBI group, the apoptotic cells in the wogonin group were significantly reduced $\left({ }^{*} \mathrm{P}<0.05\right.$ vs. sham group; ${ }^{\#} \mathrm{P}<0.05$ vs. TBI group; Fig. $\left.5 \mathrm{~B}\right)$. Then the expression of apoptosis-related proteins were detected by western blotting, including caspase-3, Bax and Bcl-2 (Fig. 6A). The results of blotting density analysis showed that TBI increased the expression of caspase- 3 and Bax, but decreased the expression of Bcl-2 on days 1, 3 and 7 after the operation ( $\mathrm{P}<0.05$ vs. sham group; Fig. 6B-J). However, compared with the TBI group, wogonin administration significantly reduced the expression of caspase- 3 and Bax and promoted the protein expression of Bcl-2 ( ${ }^{\#} \mathrm{P}<0.05$ vs. TBI group; Fig. 6B-J). This suggested that wogonin effectively inhibited the apoptosis of hippocampal CA1 area following TBI.

Wogonin promotes PI3K/Akt signaling in the hippocampus following TBI. By administering the PI3K inhibitor LY294002, the PI3K/Akt signal in the hippocampal CA1 area was checked at $24 \mathrm{~h}$ following TBI. The results showed that TBI significantly increased the expression of p-Akt and the administration of wogonin further increased the expression of p-Akt following TBI $\left({ }^{*} \mathrm{P}<0.05\right.$ vs. sham group; ${ }^{\#} \mathrm{P}<0.05$, vs. TBI group; Fig. 7A and $\left.\mathrm{B}\right)$. However, compared with the administration of wogonin, the additional application of LY294002 reduced the level of p-Akt $\left({ }^{\$} \mathrm{P}<0.05\right.$ vs. TBI + wogonin group; Fig. $7 \mathrm{~A}$ and $\left.\mathrm{B}\right)$. This showed that the regulation of wogonin on the expression of $\mathrm{p}$-Akt is through the action of PI3K. Immunofluorescence experiments have proved that $\mathrm{p}$-Akt-positive cells colocalized mainly with NeuN-positive neurons in the hippocampus of the TBI rats, suggesting that p-Akt was induced mainly in neurons (24). The results mirror the western blot results observed in Fig. 7A and B, with a robust increase of $\mathrm{p}$-Akt immunofluorescence observed in the wogonin group, suggesting that the level of p-Akt was increased in hippocampal area, which was reduced by LY294002 pre-treatment (Fig. 7C and D). It was hypothesized that wogonin serve a key role in the repair of damage following TBI by inducing PI3K/Akt signaling.

Wogonin promotes the expression of $\mathrm{Nrf} 2$ and $\mathrm{HO}-1$ proteins through the PI3K/Akt pathway. It was considered whether wogonin regulated the expression of $\mathrm{Nrf} 2$ and $\mathrm{HO}-1$, which are considered to be involved in anti-oxidation, through PI3K/Akt signal following TBI. Western blotting results showed that the administration of wogonin increased the expression of $\mathrm{Nrf} 2$ and $\mathrm{HO}-1$ following TBI ( ${ }^{*} \mathrm{P}<0.05$ vs. sham group; ${ }^{\#} \mathrm{P}<0.05$, vs. TBI group; Fig. 8A-C). Compared with the use of wogonin, the additional administration of LY294002 inhibited the expression of Nrf2 and HO-1 induced by wogonin $\left({ }^{\$} \mathrm{P}<0.05 \mathrm{vs}\right.$. $\mathrm{TBI}+$ wogonin group; Fig. 8A-C). Furthermore, the western
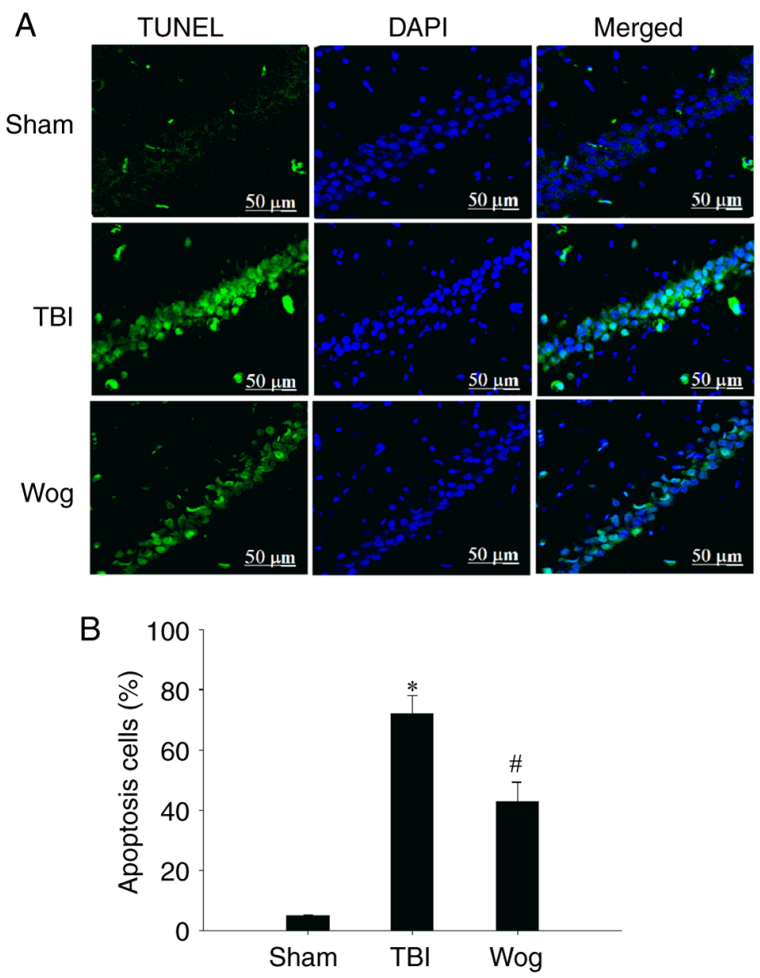

Figure 5. Wogonin inhibits hippocampal cell apoptosis induced by TBI (A) Representative confocal images of TUNEL staining were used to assess apoptosis in the sham operation group (sham), TBI model group (TBI) and wogonin administration group (Wog). TUNEL (green) and DAPI (blue) scale bar $50 \mu \mathrm{m}$. (B) Statistical analysis of apoptotic cells. Data are presented as the mean \pm standard deviation ( $\mathrm{n}=5$ /group). ${ }^{*} \mathrm{P}<0.05$ vs. sham group; ${ }^{\#} \mathrm{P}<0.05$ vs. TBI group. TBI, traumatic brain injury.

blot results were supported by double immunofluorescence for Nrf2 and NeuN, showing augmented neuronal Nrf2 immunoreactivity in the wogonin group, as compared to TBI or LY294002-treated groups at $24 \mathrm{~h}$ (Fig. 8D and E). These data proved that wogonin upregulates the antioxidant proteins $\mathrm{Nrf} 2$ and HO-1 by inducing PI3K/Akt signaling.

Wogonin reduces oxidative damage and cell apoptosis by regulating $\mathrm{PI} 3 \mathrm{~K} / \mathrm{Nrf} 2 / \mathrm{HO}-1$ pathway. Since wogonin promotes $\mathrm{PI} 3 \mathrm{~K} / \mathrm{Nrf} 2 / \mathrm{HO}-1$ signaling following TBI, whether wogonin could reduce oxidative stress and apoptosis through this pathway was verified. Western blotting results showed that the administration of wogonin inhibited the protein expression of $\mathrm{NOX}^{2}$, caspase- 3 and Bax following TBI ( $\mathrm{P}<0.05$ vs. sham group; " $\mathrm{P}<0.05$, vs. TBI group; Fig. 9A). Compared with the use of wogonin, the additional administration of LY294002 restored the levels of $\mathrm{NOX}^{2}$, caspase-3 and Bax ( ${ }^{\$} \mathrm{P}<0.05$ vs. $\mathrm{TBI}+$ wogonin group; Fig. 9B-D). For Bcl-2, the additional administration of LY294002 abolished the upregulation effect of wogonin on Bcl-2 protein expression (Fig. 9E). Therefore, the antioxidant and anti-apoptotic properties of wogonin after the occurrence of TBI are achieved by upregulating the $\mathrm{PI} 3 \mathrm{~K} / \mathrm{Akt} / \mathrm{Nrf} 2 / \mathrm{HO}-1$ signal.

\section{Discussion}

As a serious global public health problem, TBI has causes great pain and an economic burden on patients and society. 
A
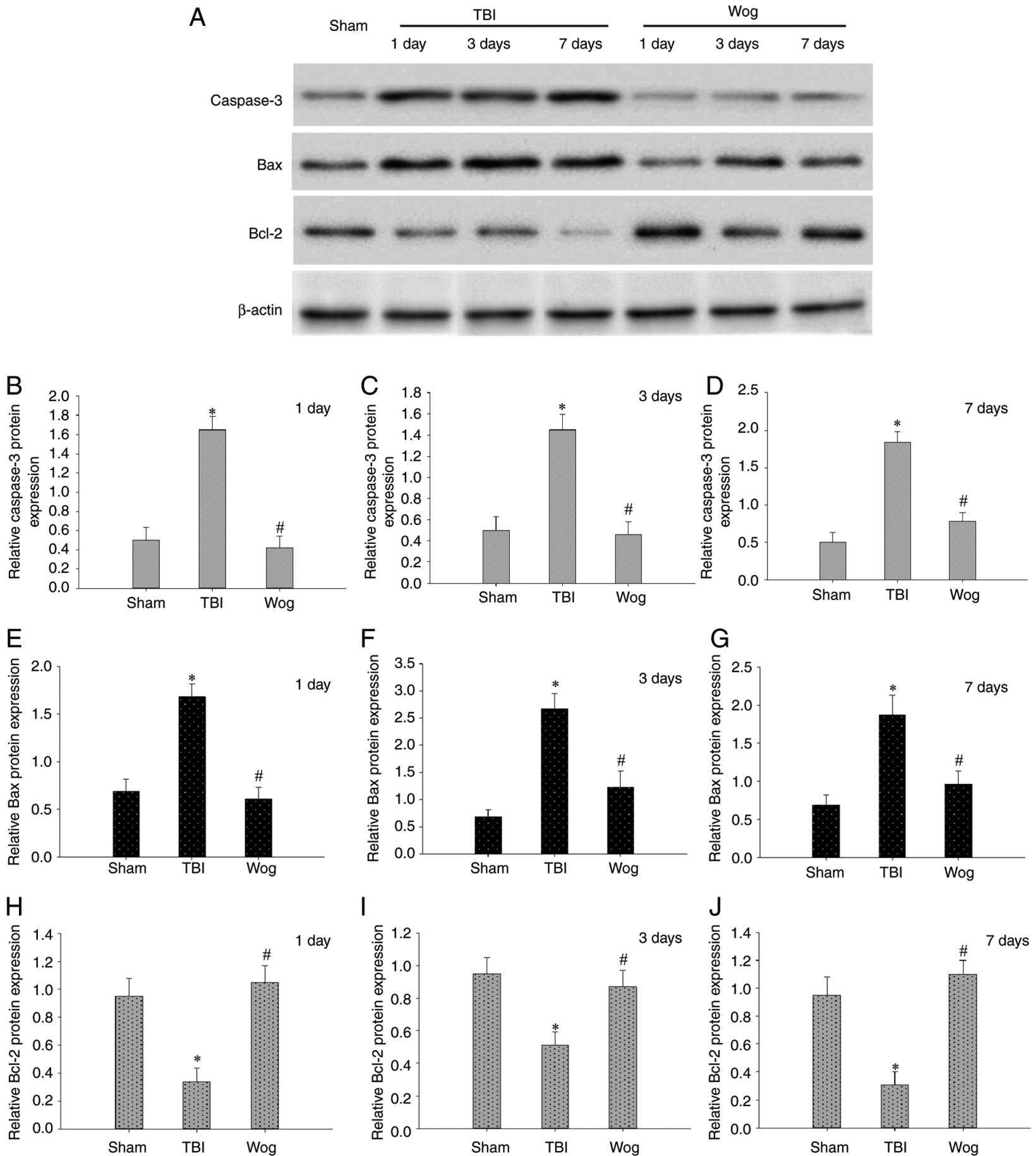

Figure 6. Wogonin regulates the level of apoptosis-related proteins in hippocampal cells. (A) Western blotting was performed on the protein levels of caspase-3, Bax and Bcl-2 in the CA1 region of the rat hippocampus of the sham operation group (sham), TBI model group (TBI) and wogonin administration group (Wog) on 1, 3 and 7 days after surgery. The optical density of (B-D) caspase-3, (E-G) Bax and (H-J) Bcl-2 bands relative to $\beta$-actin on days 1,3 and 7 were analyzed. Data are presented as the mean \pm standard deviation ( $\mathrm{n}=5 /$ group). ${ }^{*} \mathrm{P}<0.05$ vs. sham group; ${ }^{\prime \prime} \mathrm{P}<0.05$ vs. TBI group. TBI, traumatic brain injury.

The secondary chronic diseases that appear after the occurrence of TBI are mainly manifested as nerve cell death and nervous system disorders, accompanied by cerebral ischemia and even neurodegenerative diseases $(25,26)$. Although some supportive therapies have been used clinically, including anti-inflammatory, preventing thrombosis and reducing intracranial pressure, they remain insufficient to restore normal brain function in patients following TBI $(27,28)$. Therefore, it is necessary to explore the pathogenesis of TBI and the means to protect the brain tissue from damage.
Flavonoids isolated from the traditional Chinese medicine Scutellaria baicalensis, including baicalin, baicalein and wogonin, serve anti-oxidant, anti-apoptosis and tissue repair effects in a variety of diseases (29). The direct cause of tissue damage following TBI is the increase of local oxygen free radicals and the occurrence of stress oxidation reaction. Among them, ROS and MDA are directly involved in brain damage and excessive release of excitatory neurotransmitters (30). Following TBI, the levels of ROS and MDA rise sharply, while the activities of biological enzymes that 
A

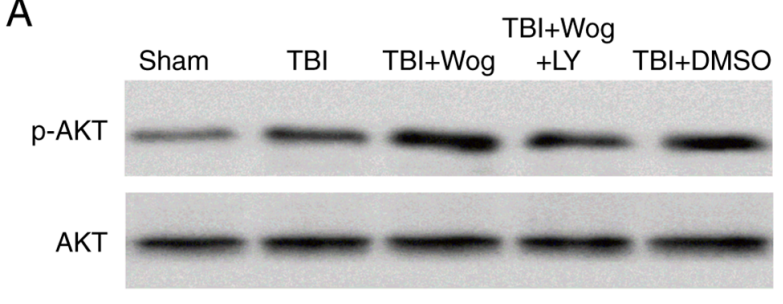

C

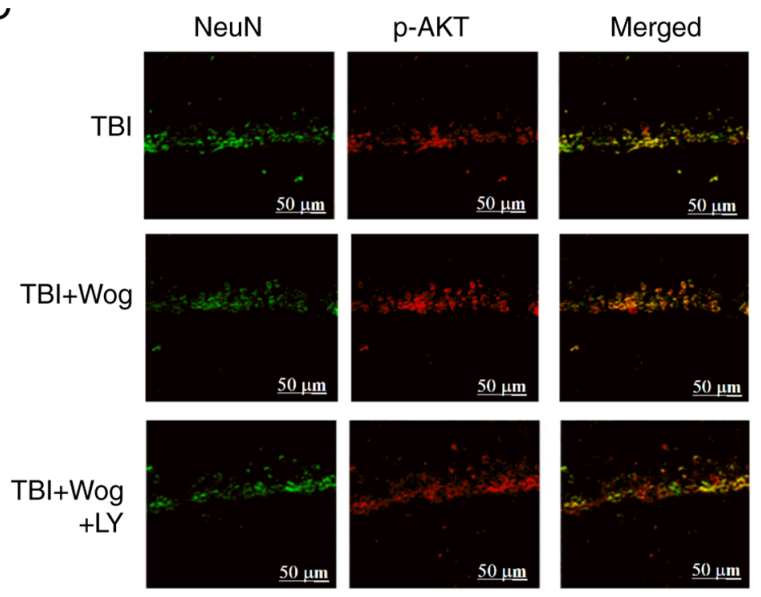

B
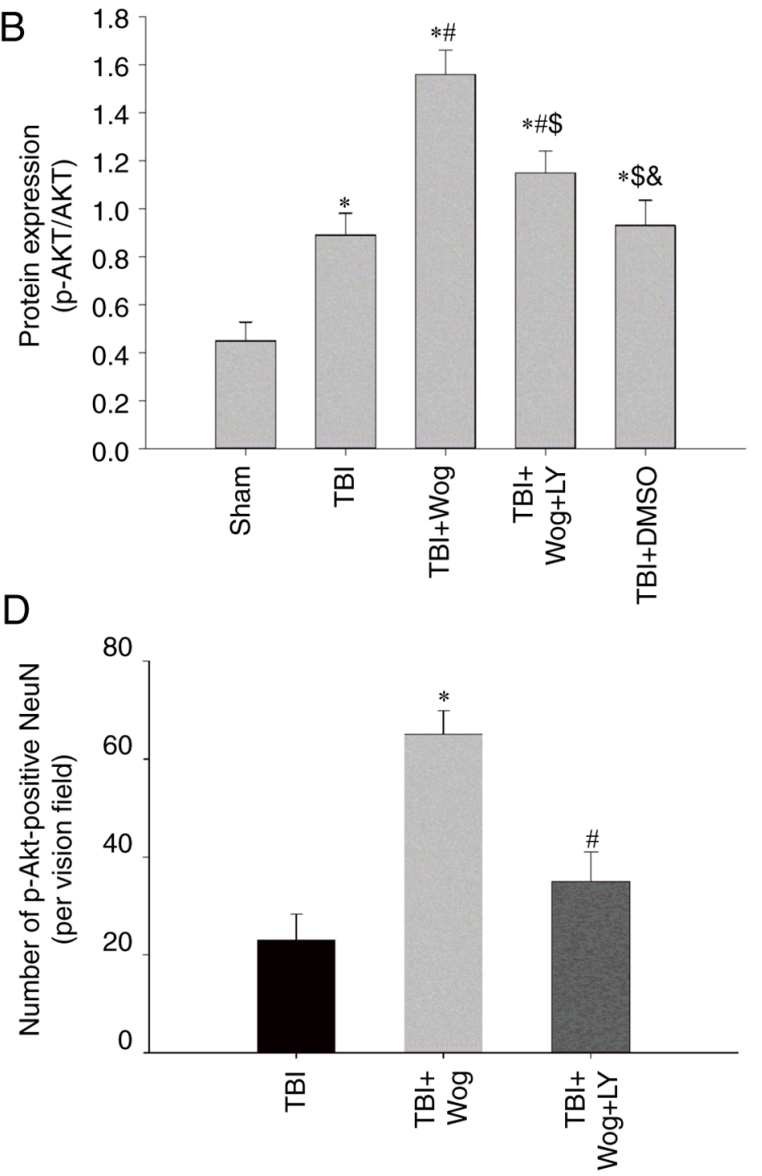

Figure 7. Wogonin promotes the expression of p-Akt following TBI in the hippocampus through PI3K. (A) western blotting was performed on the levels of p-Akt and Akt in the hippocampal CA1 region of the sham group, TBI group, wogonin administration group (TBI + Wog), wogonin and LY294002 administration group (TBI + Wog + LY) and DMSO control group (TBI + DMSO) at $24 \mathrm{~h}$ after the operation. (B) The relative optical density of the p-Akt band was analyzed. (C) Representative immunofluorescence confocal images were shown to evaluate the colocalization of p-Akt (green) and NeuN (red). (D) The quantitative analysis of p-Akt-positive NeuN in the hippocampus. Scale bar, $50 \mu \mathrm{m} .{ }^{*} \mathrm{P}<0.05$ vs. sham group; ${ }^{*} \mathrm{P}<0.05$ vs. TBI group; ${ }^{\$} \mathrm{P}<0.05 \mathrm{vs}$. TBI $+\mathrm{Wog}$ group; ${ }^{\star} \mathrm{P}<\mathrm{vs}$. TBI + Wog + LY group. p-, phosphorylated; TBI, traumatic brain injury.

scavenger oxygen free radicals, such as SOD and CAT, remain basically unchanged $(31,32)$. Excessive oxygen free radicals act on tight junction proteins, destroy the blood-brain barrier and cause brain tissue edema (33). The present study showed that TBI promoted the levels of ROS and MDA in rat hippocampus and induced cerebral edema. The main physiological functions of GSH are scavenging free radicals, anti-oxidation and anti-aging. The present study also found that plasma levels of GSH was decreased significantly in $\mathrm{ICH}$ patients from days 1-7, however, this trend was not statistically significant (34). The results of the present study also showed that GSH levels declined gradually over time in the TBI group; notably, there was no statistically significant comparison between the groups. Wogonin exerted an anti-oxidative stress effect here. It increased the activity of GSH, SOD and CAT and removed excess oxygen free radicals in the hippocampus to protect neurological damage following TBI.

Oxidative stress factors further activates the caspase family following TBI to initiate programmed death (apoptosis). After TBI, the expression of pro-apoptosis-related proteins in the injured brain tissue increases (35). In fact, there is an imbalance between anti-apoptosis and pro-apoptosis in the occurrence of apoptosis following TBI. Anti-apoptotic proteins, such as the P53 and Bcl-2 family, have been found to increase their expression following TBI $(36,37)$. Pro-apoptotic factors, mainly Bax, Bad and Caspase family proteins, whose expression increased in the lesion and surrounding brain tissues can inhibit apoptosis following TBI (38). Data from the present study confirmed that wogonin act as a neuroprotective drug to increase Bcl-2 and decrease the expression of caspase 3 and Bax. NADPH oxidase $\mathrm{NOX}^{2}$ is highly expressed in hippocampal neurons following TBI and anti-NOX ${ }^{2}$ strategies help alleviate the damage following TBI $(39,40)$. Therefore, the level of $\mathrm{NOX}^{2}$ protein in the rat TBI model was used as an indicator of oxidative stress. The present study revealed that $\mathrm{NOX}^{2}$ was increased in hippocampus neurons following TBI by both IHC staining and western blot analysis of NOX $^{2}$ protein levels of $\mathrm{NOX}^{2}$ protein between 1-7 days following TBI. Wogonin treatment markedly attenuated NOX activity and protein levels that would normally be induced by TBI. These results support the use of specific NADPH oxidase inhibitors in the treatment of TBI. Mitochondrial pathways including oxidative stress, apoptosis and $\mathrm{NOX}^{2}$ may be the targets of wogonin to inhibit neurological damage.

The PI3K/Akt signaling pathway inhibits cell apoptosis by influencing multiple downstream effector molecules (41). The neuroprotective mechanism of PI3K/Akt involves a variety of intracellular signals and effector proteins, which mainly act on 

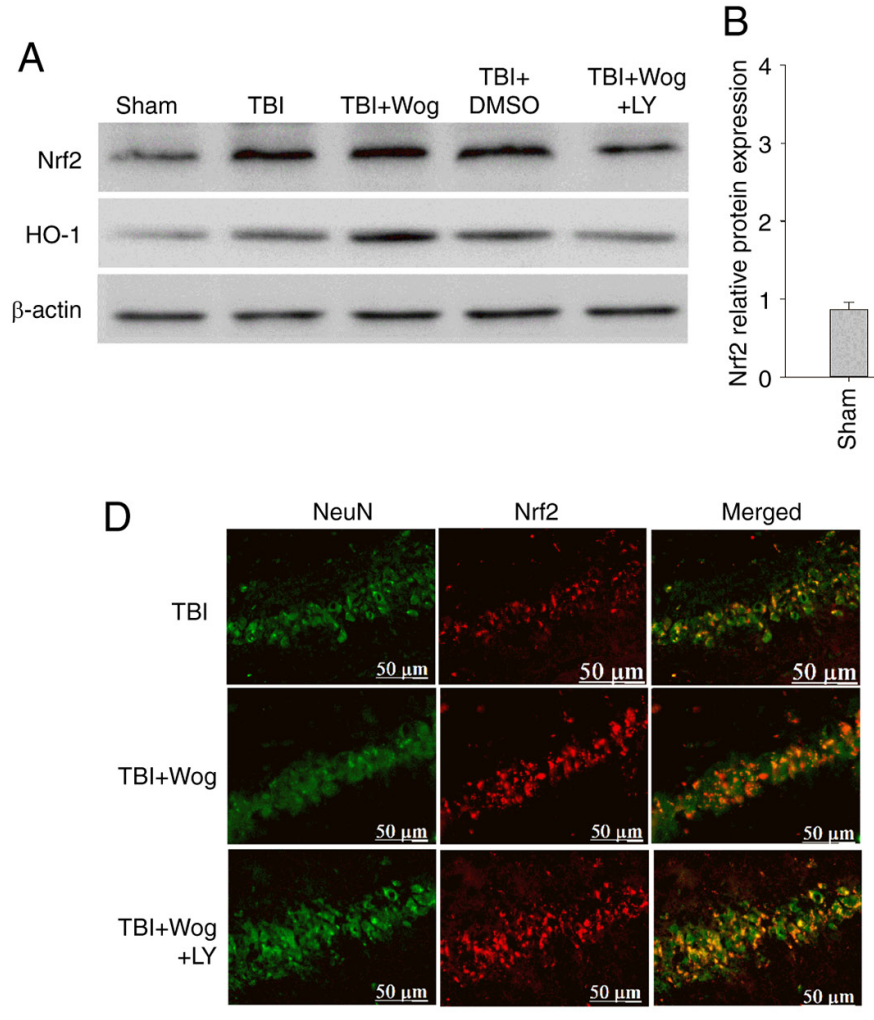

B
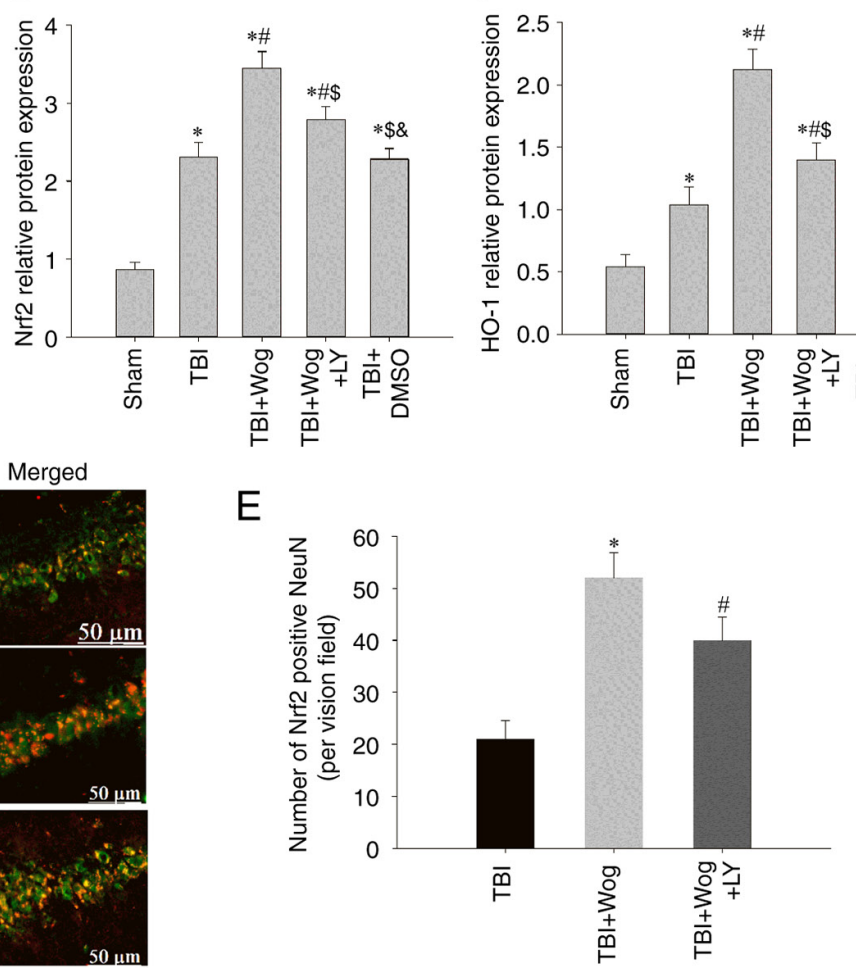

C

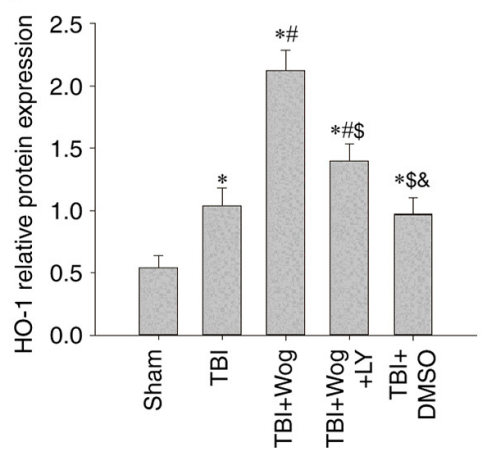

Figure 8. Wogonin increases the protein levels of Nrf2 and HO-1 following TBI in the hippocampus through the PI3K/Akt pathway. (A) Western blotting assays of Nrf2 and HO-1 were performed in the hippocampal CA1 region of the sham group, TBI group, TBI + Wog group, TBI + Wog + LY group and TBI + DMSO group at $24 \mathrm{~h}$ after the operation. The optical density of (B) Nrf2 and (C) HO-1 bands relative to $\beta$-actin was analyzed. (D) Representative immunofluorescence confocal images were used to evaluate the co-localization of Nrf2 (green) and NeuN (red). (E) The quantitative analysis of Nrf2-positive NeuN in the hippocampus. Scale bar, $50 \mu \mathrm{m}$. ${ }^{*} \mathrm{P}<0.05$ vs. sham group; ${ }^{\prime \prime} \mathrm{P}<0.05$ vs. TBI group; ${ }^{\$} \mathrm{P}<0.05$ vs. TBI + Wog group; ${ }^{\circledR} \mathrm{P}<\mathrm{vs}$. TBI + Wog $+\mathrm{LY}$ group. Nrf2, nuclear factor-erythroid factor 2-related factor 2; HO-1, heme oxygenase-1; TBI, traumatic brain injury.

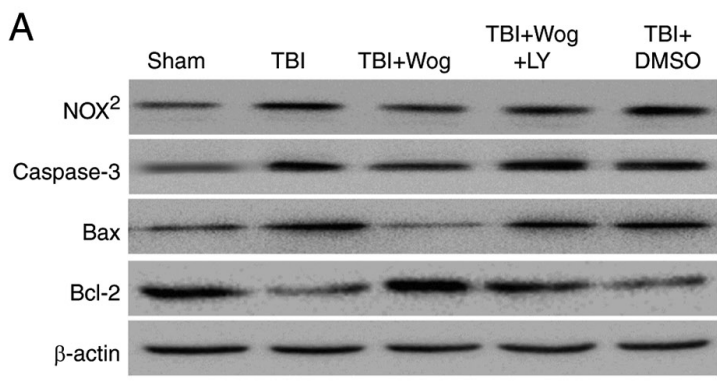

C

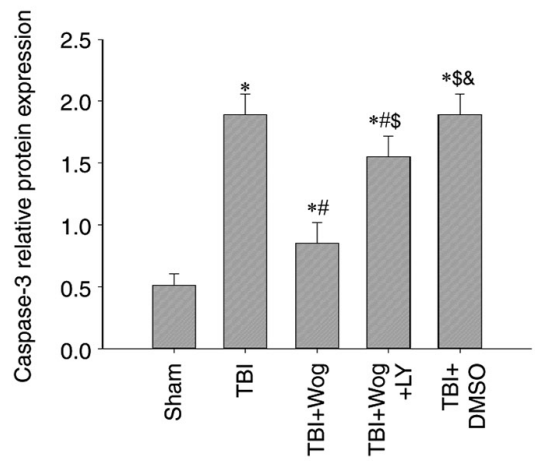

D

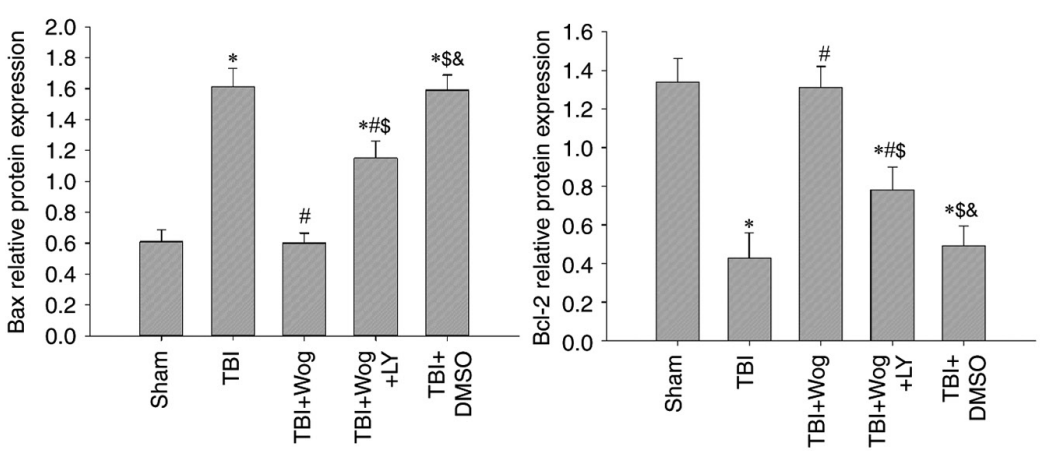

Figure 9. Wogonin reduces oxidative damage and cell apoptosis by regulating the PI3K/Nrf2/HO-1 pathway. (A) Western blotting analysis showed NOX ${ }^{2}$, caspase-3, Bax and Bcl-2 protein levels in the rat hippocampus at $24 \mathrm{~h}$ from the sham group, TBI group, TBI + Wog group, TBI + Wog + LY group and TBI + DMSO group. (B-E) The optical density of $\mathrm{NOX}^{2}$, caspase-3, Bax and Bcl-2 bands relative to $\beta$-actin was analyzed. ${ }^{\mathrm{P}}<0.05$ vs. sham group; ${ }^{*} \mathrm{P}<0.05$ vs. TBI group; ${ }^{\mathrm{s}} \mathrm{P}<0.05$ vs. TBI + Wog group; ${ }^{\&} \mathrm{P}<$ vs. TBI + Wog + LY group. Nrf2, nuclear factor-erythroid factor 2-related factor 2; HO-1, heme oxygenase-1; TBI, traumatic brain injury. 


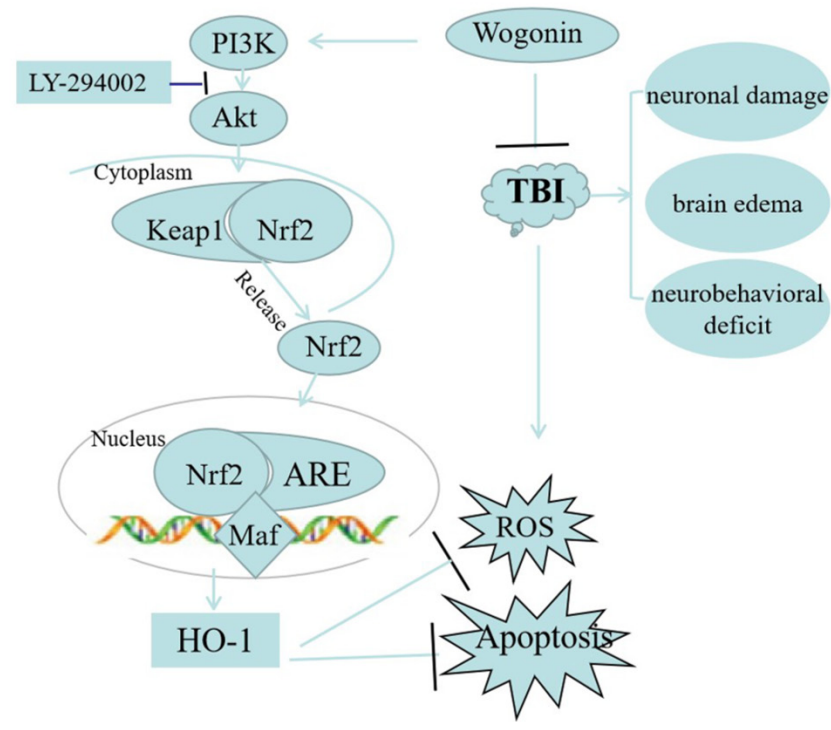

Figure 10. Schematic diagram of the neuroprotective mechanism of wogonin after the occurrence of TBI. Wogonin increases the level of phosphorylated AKT and activates Nrf2, which translocates into the nucleus to increase HO-1 production. The high expression of HO-1 is responsible for anti-apoptosis and anti-oxidative stress, realizing the repair of TBI. TBI, traumatic brain injury; Nrf2, nuclear factor-erythroid factor 2-related factor 2; HO-1, heme oxygenase-1; Keap1, Kelch-like ECH-associated protein; ROS, reactive oxygen species.

downstream targets through activated phosphorylated Akt to exert anti-apoptotic effects. PI3K/Akt signaling pathway can be activated by drugs and non-drug means, including wogonin, to promote the survival of neurons and the protection of TBI (24). For example, atorvastatin inhibits the expression of miR-126 and then upregulates the PI3K/AKT signaling pathway to promote angiogenesis in the brain tissue of TBI rats (42). In addition, scriptaid, a deacetylase HDAC inhibitor, enhances PTEN/PI3K/Akt signaling and restores microglia function after severe TBI (43). The adrenergic receptor agonist dexmedetomidine activates the PI3K/Akt/mTOR signaling pathway and exerts neuroprotective effects in TBI rats (44). The present study revealed that the activation of PI3K/Akt signaling pathways was beneficial for wogonin to exert anti-oxidative effects and to consequently protect against brain injury. The administration of wogonin significantly promoted the phosphorylation of Akt and contributed to the anti-oxidation of hippocampus following TBI. In fact, the effect of wogonin on the PI3K/Akt pathway has been previously implied in some other diseases. The mechanism of wogonin in the treatment of lung cancer involves the PI3K/Akt signaling pathway (45). Wogonin reduces renal tubular damage by regulating autophagy and inflammation mediated by PI3K/Akt/NF- $\kappa \mathrm{B}$ signaling pathway (13). For nervous system and brain diseases, wogonin has been found to inhibit the phosphorylation of Akt and NF- $\mathrm{BB}$ and delay the growth of gliomas (46). The present study is the first, to the best of the authors' knowledge, to reveal the role of wogonin in promoting neurological function following TBI by regulating the PI3K/Akt pathway. Following the administration of PI3K inhibitor LY294002, the antioxidant and anti-apoptotic effects of wogonin on the hippocampus of rats following TBI were greatly reduced. This inhibitory effect by LY294002 indicated that the function of wogonin depended on the PI3K/Akt pathway. LY294002 has also been used in other studies to reveal the role of wogonin. For example, LY294002 in breast cancer MCF-7 cells increased wogonin-induced apoptosis through PI3K/Akt/survivin signaling pathway blockade (47). Studies have also proved that LY294002 destroys the blood-brain barrier and causes neurological dysfunction and cognitive impairment following TBI $(48,49)$. Therefore, in the follow-up routine or drug treatment of TBI patients, it is necessary to avoid mixing the inhibitory components of PI3K/Akt.

Nrf2 plays an important role in the endogenous antioxidant mechanism following TBI. In the physiological state, Nrf2 in the cytoplasm binds to the actin binding protein Kelch-like ECH-associated protein 1 (Keap1) and the activity of Nrf2 is inhibited. Under TBI pathological stimulation, $\mathrm{Nrf} 2$ is phosphorylated and uncoupled from Keap1 (50). The active Nrf2 is transferred into the nucleus and the ARE/MAF response element is combined to initiate the expression of ARE-regulated antioxidant enzyme genes, such as HO-1 (51). HO-1 is regulated by Nrf2 and exerts anti-oxidant, anti-inflammatory and anti-apoptotic effects in different in vitro and in vivo models (52). A study showed that in $\mathrm{Nrf} 2$ gene-deficient mice, brain damage following TBI increases, manifesting as neuroinflammation and apoptosis (53). There have been some drugs or methods used to alleviate TBI-related neurological disorders by targeting Nrf2 and HO-1. For example, the D2-like receptor agonist pramipexole activates the Nrf2/HO-1 signaling pathway to serve a neuroprotective effect following TBI (9). The behavioral changes and reduction of oxidative stress in Wistar rats treated with tannin following TBI were attributed to the activation of PGC- $1 \alpha / \mathrm{Nrf}-2 / \mathrm{HO}-1$ signaling pathway (54). Some natural compound monomers, including wogonin, also regulate $\mathrm{Nrf} 2 / \mathrm{HO}-1$ signaling to relieve $\mathrm{TBI}$ symptoms. Astaxanthin, a carotenoid pigment, upregulates the expression of Nrf2 and HO-1 following TBI and provides neurological protection (55). Breviscapine treatment from Erigeron breviscapus upregulates the expression of $\mathrm{Nrf} 2$ and HO-1, reducing TBI-induced neuronal cell apoptosis and improving neurobehavioral function (56). The present study revealed that wogonin participated in neuroprotection in the hippocampus by increasing Nrf2/HO-1 signaling. Wogonin's regulation of $\mathrm{Nrf} 2 / \mathrm{HO}-1$ expression in the hippocampus following TBI was dependent on PI3K/Akt, because the administration of LY294002 rescued the biological phenomenon. Although some previous studies have proved that the PI3K/Akt-mediated Nrf2/HO-1 signaling pathway directly protects a number of types of cells from oxidative stress and apoptosis (57-59), the mechanism following TBI is not fully understood. The present study revealed the molecular mechanism of Nrf2/HO-1 activated by PI3K/Akt on neurological dysfunction following TBI and proposed the therapeutic effect of wogonin in it.

In summary, the present study proposed a neuroprotective effect and mechanism for wogonin in TBI (Fig. 10). Wogonin improved neurological deficits and learning and memory abilities and relieved cerebral edema following TBI. In mechanism, administration of wogonin reduced the oxidative stress and apoptosis of nerve tissues, which was attributed to the activation of PI3K/Akt/Nrf2/HO-1 pathway. The present study revealed the role and mechanism of wogonin in protecting secondary damage following TBI. 


\section{Acknowledgements}

Not applicable.

\section{Funding}

The present study was financially supported by the National Natural Science Foundation of China (grant no. 81401032), the Natural Science Foundation of Hebei Province (grant no. H2020206437), the Medical Science Research Project of Hebei Province (grant no. 20190063) and the Hebei Medical University Department-School Consultation Fund-Science and Technology Innovation-Frontier Cross Discipline Research (grant no. 2020TXJC02).

\section{Availability of data and materials}

All data generated or analyzed during this study are included in this published article.

\section{Authors' contributions}

YF and YJ performed the experiments. ZY and MJ assisted in performing the brain water measurement, MWM and NSS tests. QW and MY assisted in performing the H\&E and immunofluorescent staining and western blotting. GS was responsible for designing the study, summarizing the results and writing the manuscript. LW participated in data analysis and involved in drafting the manuscript or revising it critically for important intellectual content. YF and GS confirm the authenticity of all the raw data. All authors read and approved the final manuscript.

\section{Ethics approval and consent to participate}

All animal experiments were approved by the Ethics Committee of Hebei Medical University (permit no. 20201086). Humane endpoints were established following the guideline of assessment for humane endpoints in animal experiment (People's Republic of China: RB/T 173-2018) in order to minimize pain or distress to experimental animals.

\section{Patient consent for publication}

Not applicable

\section{Competing interests}

The authors declare that they have no competing int

\section{References}

1. Chowdhury T, Kowalski S, Arabi Y and Dash HH: Specific intensive care management of patients with traumatic brain injury: Present and future. Saudi J Anaesth 8: 268-275, 2014.

2. Vidhya V, Gudigar A, Raghavendra U, Hegde A, Menon GR, Molinari F, Ciaccio EJ and Acharya UR: Automated detection and screening of traumatic brain injury (TBI) using computed tomography images: A comprehensive review and future perspectives. Int J Environ Res Public Health 18: 6499, 2021.

3. Schweitzer AD, Niogi SN, Whitlow CJ and Tsiouris AJ: Traumatic brain injury: Imaging patterns and complications. Radiographics 39: 1571-1595, 2019.
4. Yu LM, Dong X, Xue XD, Zhang J, Li Z, Wu HJ, Yang ZL, Yang Y and Wang HS: Naringenin improves mitochondrial function and reduces cardiac damage following ischemia-reperfusion injury: The role of the AMPK-SIRT3 signaling pathway. Food Funct 10: 2752-2765, 2019.

5. Qian F, Han Y, Han Z, Zhang D, Zhang L, Zhao G, Li S, Jin G, Yu R and Liu H: In situ implantable, post-trauma microenvironment-responsive, ROS depletion hydrogels for the treatment of traumatic brain injury. Biomaterials 270: 120675, 2021.

6. Slavoaca D, Muresanu D, Birle C, Rosu OV, Chirila I, Dobra I, Jemna N, Strilciuc S and Vos P: Biomarkers in traumatic brain injury: New concepts. Neurolog Sci 41: 2033-2044, 2020.

7. Bedard $\mathrm{K}$ and Krause KH: The NOX family of ROS-generating NADPH oxidases: Physiology and pathophysiology. Physiol Rev 87: 245-313, 2007.

8. Chandran R, Kim T, Mehta SL, Udho E, Chanana V, Cengiz P, Kim H, Kim C and Vemuganti R: A combination antioxidant therapy to inhibit $\mathrm{NOX}^{2}$ and activate Nrf2 decreases secondary brain damage and improves functional recovery after traumatic brain injury. J Cereb Blood Flow Metab 38: 1818-1827, 2018.

9. Salman M, Tabassum $\mathrm{H}$ and Parvez S: Nrf2/HO-1 mediates the neuroprotective effects of pramipexole by attenuating oxidative damage and mitochondrial perturbation after traumatic brain injury in rats. Dis Model Mech 13: dmm045021, 2020.

10. Hassanin M, Mai T, Tadros M, Elmazar M and Singab AN: Wogonin a promising component of scutellaria baicalensis: A review on its chemistry, pharmacokinetics and biological activities. Archives of Pharmaceutical Sciences Ain Shams University 3: 170-179, 2019.

11. Huynh DL, Ngau TH, Nguyen NH, Tran BG and Nguyen CT: Potential therapeutic and pharmacological effects of Wogonin: An updated review. Mol Biol Rep 47: 9779-9789, 2020.

12. Chun W, Lee HJ, Kong PJ, Lee GH, Cheongsu LY, Park H and Kim SS: Synthetic wogonin derivatives suppress lipopolysaccharide-induced nitric oxide production and hydrogen peroxide-induced cytotoxicity. Arch Pharm Res 28: 216-219, 2005.

13. Lei L, Zhao J, Liu XQ, Chen J, Qi XM, Xia LL and Wu YG: Wogonin alleviates kidney tubular epithelial injury in diabetic nephropathy by inhibiting PI3K/Akt/NF- $\mathrm{BB}$ signaling pathways. Drug Des Devel Ther 15: 3131-3150, 2021.

14. Zhu G, Zhang J, Yang Y, Zhang H, Jin W, Su F, Liang J, Wang K, Zhang $\mathrm{J}$ and Chen C: The key target and molecular mechanism of the volatile component of scutellaria baicalensis georgi in acute lung injury based on network pharmacology. Front Pharmacol 12: 650780, 2021.

15. Umemoto Y, Patel A, Huynh T and Chitravanshi VC: Wogonin attenuates the deleterious effects of traumatic brain injury in anesthetized Wistar rats. Eur J Pharmacol 848: 121-130, 2019.

16. Chen CC, Hung TH, Wang YH, Lin CW, Wang PY, Lee CY and Chen SF: Wogonin improves histological and functional outcomes, and reduces activation of TLR $4 / \mathrm{NF}-\kappa \mathrm{B}$ signaling after experimental traumatic brain injury. PLoS One 7: e30294, 2012.

17. Feng Y, Gao J, Cui Y, Li M, Li R, Cui C and Cui J: Neuroprotective effects of resatorvid against traumatic brain injury in rat: Involvement of neuronal autophagy and TLR4 signaling pathway. Cell Mol Neurobiol 37: 155-168, 2017.

18. Ates O, Cayli S, Altinoz E, Gurses I, Yucel N, Sener M, Kocak A and Yologlu S: Neuroprotection by resveratrol against traumatic brain injury in rats. Mol Cell Biochem 294: 137-144, 2007.

19. Chen SF, Hsu CW, Huang WH and Wang JY: Post-injury baicalein improves histological and functional outcomes and reduces inflammatory cytokines after experimental traumatic brain injury. Br J Pharmacol 155: 1279-1296, 2008.

20. Feng Y, Cui Y, Gao JL, Li MH, Li R, Jiang XH, Tian YX, Wang KJ, Cui CM and Cui JZ: Resveratrol attenuates neuronal autophagy and inflammatory injury by inhibiting the TLR4/NF- $\kappa \mathrm{B}$ signaling pathway in experimental traumatic brain injury. Int J Mol Med 37: 921-930, 2016.

21. Changmeng C, Sixin S, Jianzhong C, Yan F, Junling G and Pei J: Vitamin D receptor activation influences NADPH oxidase $\left(\mathrm{NOX}^{2}\right)$ activity and protects against neurological deficits and apoptosis in a rat model of traumatic brain injury. Oxid Med Cell Longev 2017: 9245702, 2017.

22. Wang P, Wu Q, Wu W, Li H, Guo Y, Yu P, Gao G, Shi Z, Zhao B and Chang YZ: Mitochondrial ferritin deletion exacerbates $\beta$-amyloid-induced neurotoxicity in mice. Oxid Med Cell Longev 2017: 1020357, 2017. 
23. Song SX, Gao JL, Wang KJ, Li R, Tian YX, Wei JQ and Cui JZ: Attenuation of brain edema and spatial learning deficits by the inhibition of NADPH oxidase activity using apocynin following diffuse traumatic brain injury in rats. Mol Med Rep 7: 327-331, 2013.

24. Du G, Zhao Z, Chen Y, Li Z, Tian Y, Liu Z, Liu B and Song J: Quercetin attenuates neuronal autophagy and apoptosis in rat traumatic brain injury model via activation of PI3K/Akt signaling pathway. Neurol Res 38: 1012-1019, 2016.

25. Netteland DF, Mejlnder-Evjensvold M, Skaga NO, Sandset EC, Aarhus $M$ and Helseth E: Cerebral venous thrombosis in traumatic brain injury: A cause of secondary insults and added mortality. J Neurosurg 134: 1-9, 2020

26. Robicsek SA, Bhattacharya A, Rabai F, Shukla K and Doré S: Blood-related toxicity after traumatic brain injury: Potential targets for neuroprotection. Mol Neurobiol 57: 159-178, 2020.

27. Khellaf A, Khan DZ and Helmy A: Recent advances in traumatic brain injury. J Neurol 266: 2878-2889, 2019.

28. Gennai S, Monsel A, Hao Q, Liu J, Gudapati V, Barbier EL and Lee JW: Cell-based therapy for traumatic brain injury. $\mathrm{Br}$ J Anaesth 115: 203-212, 2015.

29. Huynh DL, Sharma N, Singh AK, Sodhi SS, Zhang JJ, Mongre RK, Ghosh M, Kim N, Park YH and Jeong DK: Anti-tumor activity of wogonin, an extract from Scutellaria baicalensis, through regulating different signaling pathways. Chin J Nat Med 15: 15-40, 2017.

30. Du D, Tang W, Zhou C, Sun X, Wei Z, Zhong J and Huang Z: Fecal microbiota transplantation is a promising method to restore gut microbiota dysbiosis and relieve neurological deficits after traumatic brain injury. Oxid Med Cell Longev 2021: 5816837, 2021.

31. Ji X, Tian Y, Xie K, Liu W, Qu Y and Fei Z: Protective effects of hydrogen-rich saline in a rat model of traumatic brain injury via reducing oxidative stress. J Surg Res 178: e9-e16, 2012.

32. Liu C, He D and Zhao Q: Licoricidin improves neurological dysfunction after traumatic brain injury in mice via regulating FoxO3/wnt/ $\beta$-catenin pathway. J Nat Med 74: 767-776, 2020.

33. Chen J, Chen W, Han K, Qi E, Chen R, Yu M, Hou L and Lv L: Effect of oxidative stress in rostral ventrolateral medulla on sympathetic hyperactivity after traumatic brain injury. Eur J Neurosci 50: 1972-1980, 2019.

34. Masomi-Bornwasser J, Kurz E, Frenz C, Schmitt J, Wesp DMA, König J, Lotz J, Ringel F, Kerz T, Krenzlin H and Keric N: The influence of oxidative stress on neurological outcomes in spontaneous intracerebral hemorrhage. Biomolecules 11: 1615, 2021.

35. Carteri RB, Kopczynski A, Rodolphi MS, Strogulski NR, Sartor M, Feldmann M, Bastiani MAD, Wannmacher CMD, de Franceschi ID, Hansel G, et al: Testosterone administration after traumatic brain injury reduces mitochondrial dysfunction and neurodegeneration. J Neurotrauma 36: 2246-2259, 2019.

36. Hong MY, Gao JL, Cui JZ, Wang KJ, Tian YX, Li R, Wang HT and Wang H: Effect of c-Jun NH-terminal kinase-mediated p53 expression on neuron autophagy following traumatic brain injury in rats. Chinese Med J (Engl) 125: 2019-2024, 2012.

37. Deng H, Yue JK, Zusman BE, Nwachuku EL, Abou-Al-Shaar H, Upadhyayula PS, Okonkwo DO and Puccio AM: B-cell lymphoma $2(\mathrm{Bcl}-2)$ and regulation of apoptosis after traumatic brain injury: A clinical perspective. Medicina (Kaunas) 56: 300, 2020.

38. Schober ME, Requena DF, Block B, Davis LJ, Rodesch C, Casper TC, Juul SE, Kesner RP and Lane RH: Erythropoietin improved cognitive function and decreased hippocampal caspase activity in rat pups after traumatic brain injury. J Neurotrauma 31: 358-369, 2014

39. Jackman KA, Miller AA, de Silva TM, Crack PJ, Drummond GR and Sobey CG: Reduction of cerebral infarct volume by apocynin requires pretreatment and is absent in Nox2-defificient mice. $\mathrm{Br}$ J Pharmacol 156: 680-688, 2009.

40. Dohi K, Ohtaki H, Nakamachi T, Yofu S, Satoh K, Miyamoto K, Song D, Tsunawaki S, Shioda S and Aruga T: Gp91phox (NOX2) in classically activated microglia exacerbates traumatic brain injury. J Neuroinflflammation 7: 41, 2010.

41. Wang M, Zhang J and Gong N: Role of the PI3K/Akt signaling pathway in liver ischemia reperfusion injury: A narrative review. Ann Palliat Med 27: apm-21-3286, 2021.

42. Li Q, Cheng K, Wang AY, Xu QG, Fu ZF, He SY and Xu PX: microRNA-126 inhibits tube formation of HUVECs by interacting with EGFL7 and down-regulating PI3K/AKT signaling pathway. Biomed Pharmacother 116: 109007, 2019.
43. Wang G, Shi Y, Jiang X, Leak RK, Hu X, Wu Y, Pu H, Li WW, Tang B, Wang Y, et al: HDAC inhibition prevents white matter injury by modulating microglia/macrophage polarization through the GSK3 $3 /$ PTEN/Akt axis. Proc Natl Acad Sci USA 112: 2853-2858, 2015 .

44. Shen M, Wang S, Wen X, Han XR, Wang YJ, Zhou XM, Zhang MH, Wu DM, Lu J and Zheng YL: Dexmedetomidine exerts neuroprotective effect via the activation of the $\mathrm{PI} 3 \mathrm{~K} / \mathrm{Akt} / \mathrm{mTOR}$ signaling pathway in rats with traumatic brain injury. Biomed Pharmacother 95: 885-893, 2017.

45. Wang L, Zhang J, Shan G, Liang J, Jin W, Li Y, Su F, Ba Y, Tian X, Sun X, et al: Establishment of a lung cancer discriminative model based on an optimized support vector machine algorithm and study of key targets of Wogonin in lung cancer. Front Pharmacol 12: 728937, 2021

46. Parajuli P, Joshee N, Chinni SR, Rimando AM, Mittal S, Sethi S and Yadav AK: Delayed growth of glioma by Scutellaria flavonoids involve inhibition of Akt, GSK-3 and NF- $\kappa$ B signaling. J Neurooncol 101: 15-24, 2011

47. Huang KF, Zhang GD, Huang YQ and Diao Y: Wogonin induces apoptosis and down-regulates survivin in human breast cancer MCF-7 cells by modulating PI3K-AKT pathway. Int Immunopharmacol 12: 334-341, 2012.

48. Wang ZG, Cheng Y, Yu XC, Ye LB, Xia QH, Johnson NR, Wei X, Chen DQ, Cao G, Fu XB, et al: bFGF protects against blood-brain barrier damage through junction protein regulation via PI3K-Akt-Rac1 pathway following traumatic brain injury. Mol Neurobiol 53: 7298-7311, 2016.

49. Wu F, Chen Z, Tang C, Zhang J, Cheng L, Zuo H, Zhang H, Chen D, Xiang L, Xiao J, et al: Acid fibroblast growth factor preserves blood-brain barrier integrity by activating the PI3K-Akt-Rac1 pathway and inhibiting RhoA following traumatic brain injury. Am J Transl Res 9: 910-925, 2017.

50. Liao K, Su X, Lei K, Liu Z, Lu L, Wu Q, Pan H, Huang Q, Zhao Y, Wang M, et al: Sinomenine protects bone from destruction to ameliorate arthritis via activating p62Thr269/Ser272-Keap1-Nrf2 feedback loop. Biomed Pharmacother 135: 111195, 2021.

51. Motohashi H, Katsuoka F, Engel JD and Yamamoto M: Small maf proteins are obligate transcriptional cofactors for normal keratinocyte differentiation in the Keap1-Nrf2 regulatory pathway. Proc Natl Acad Sci USA 101: 6379-6384, 2004.

52. Ndisang JF: Synergistic interaction between heme oxygenase (HO) and nuclear-factor E2- related factor-2 (Nrf2) against oxidative stress in cardiovascular related diseases. Curr Pharm Des 23: 1465-1470, 2017.

53. Bhowmick S, D'Mello V, Caruso D and Abdul-Muneer PM: Traumatic brain injury-induced downregulation of Nrf2 activates inflammatory response and apoptotic cell death. J Mol Med (Berl) 97: 1627-1641, 2019.

54. Salman M, Tabassum H and Parvez S: Tannic acid provides neuroprotective effects against traumatic brain injury through the PGC-1 $\alpha /$ Nrf2/HO-1 pathway. Mol Neurobiol 57: 2870-2885, 2020.

55. Gao F, Wu X, Mao X, Niu F, Zhang B, Dong J and Liu B: Astaxanthin provides neuroprotection in an experimental model of traumatic brain injury via the Nrf2/HO-1 pathway. Am J Transl Res 13: 1483-1493, 2021.

56. Li F, Wang X, Zhang Z, Gao P and Zhang X: Breviscapine provides a neuroprotective effect after traumatic brain injury by modulating the Nrf2 signaling pathway. J Cell Biochem 120: 14899-14907, 2019.

57. Zhuang Y, Wu H, Wang X, He J, He S and Yin Y: Resveratrol attenuates oxidative stress-induced intestinal barrier injury through PI3K/Akt-mediated Nrf2 signaling pathway. Oxid Med Cell Longev 2019: 7591840, 2019.

58. Li ST, Dai Q, Zhang SX, Liu YJ, Yu QQ, Tan F, Lu SH, Wang Q, Chen JW, Huang HQ, et al: Ulinastatin attenuates LPS-induced inflammation in mouse macrophage RAW264.7 cells by inhibiting the JNK/NF- $\mathrm{KB}$ signaling pathway and activating the PI3K/Akt/Nrf2 pathway. Acta Pharmacol Sin 39: 1294-1304, 2018.

59. Wen Z, Hou W, Wu W, Zhao Y, Dong X, Bai X, Peng L and Song L: 6'-O-Galloylpaeoniflorin attenuates cerebral ischemia reperfusion-induced neuroinflammation and oxidative stress via PI3K/Akt/Nrf2 activation. Oxid Med Cell Long 2018: 8678267, 2018.

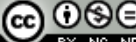

This work is licensed under a Creative Commons Attribution-NonCommercial-NoDerivatives 4.0 International (CC BY-NC-ND 4.0) License. 